\title{
CONVECTION IN CONDENSIBLE-RICH ATMOSPHERES
}

\author{
F. Ding ${ }^{1}$ and R. T. PierrehumberT ${ }^{2}$ \\ ${ }^{1}$ Department of the Geophysical Sciences, University of Chicago, Chicago, IL 60637, USA; fding@uchicago.edu \\ 2 Department of Physics, University of Oxford, Oxford OX1 3PU, UK \\ Received 2015 December 12; accepted 2016 February 27; published 2016 April 28
}

\begin{abstract}
Condensible substances are nearly ubiquitous in planetary atmospheres. For the most familiar case-water vapor in Earth's present climate - the condensible gas is dilute, in the sense that its concentration is everywhere small relative to the noncondensible background gases. A wide variety of important planetary climate problems involve nondilute condensible substances. These include planets near or undergoing a water vapor runaway and planets near the outer edge of the conventional habitable zone, for which $\mathrm{CO}_{2}$ is the condensible. Standard representations of convection in climate models rely on several approximations appropriate only to the dilute limit, while nondilute convection differs in fundamental ways from dilute convection. In this paper, a simple parameterization of convection valid in the nondilute as well as dilute limits is derived and used to discuss the basic character of nondilute convection. The energy conservation properties of the scheme are discussed in detail and are verified in radiative-convective simulations. As a further illustration of the behavior of the scheme, results for a runaway greenhouse atmosphere for both steady instellation and seasonally varying instellation corresponding to a highly eccentric orbit are presented. The latter case illustrates that the high thermal inertia associated with latent heat in nondilute atmospheres can damp out the effects of even extreme seasonal forcing.
\end{abstract}

Key words: convection - planets and satellites: atmospheres - planets and satellites: terrestrial planets

\section{INTRODUCTION}

Most planetary atmospheres contain one or more condensible substances, which can undergo phase transitions from vapor to liquid or solid form within the atmosphere. The most familiar condensible is water vapor, which plays a key role in Earth's climate and in the runaway water vapor greenhouse, which is central to the past climate evolution of Venus and also determines the inner edge of the habitable zone (Ingersoll 1969; Kasting 1988; Nakajima et al. 1992; Kasting et al. 1993; Kopparapu et al. 2013). $\mathrm{CO}_{2}$ condensation is of importance on both present and Early Mars and plays a similar role to water vapor in determining the outer edge of the habitable zone (Pierrehumbert 2011). Condensible substances are ubiquitous in planetary atmospheres; additional examples include $\mathrm{CH}_{4}$ on Titan, $\mathrm{N}_{2}$ on Triton and possibly in Titan's past climates, $\mathrm{NH}_{3}$ and $\mathrm{NH}_{4} \mathrm{SH}$ on Jupiter and Saturn, and $\mathrm{CH}_{4}$ on Uranus and Neptune. On hot Jupiters and other strongly irradiated planets condensibles can even include a variety of substances more commonly thought of as rocks and minerals in Earth-like conditions, such as enstatite $\left(\mathrm{MgSiO}_{3}\right)$ or iron. In this paper, the term "moist" will be used to refer to any condensible substance, not just water vapor. The diversity of climate phenomena associated with the choice of condensible is augmented by the variety of relevant noncondensible background gases. For example, planets with an $\mathrm{H}_{2}$ background gas are of considerable interest, and even Earth- and super-Earth-sized planets can retain an $\mathrm{H}_{2}$ atmosphere if they are in sufficiently distant orbits (Pierrehumbert \& Gaidos 2011).

In the present Earth's climate, water vapor is a dilute condensible, in the sense that water vapor makes up a small portion of any parcel of air. For example, saturated air at a tropical surface temperature of $300 \mathrm{~K}$ contains about $3.5 \%$ water vapor, measured as a molar (also called volumetric) concentration. The convection parameterizations in use in conventional terrestrial general circulation models (GCMs) rely on a number of approximations appropriate to the dilute limit.
If the planet in question has a large condensed reservoir, such as an ocean or an icy crust, then nondiluteness increases with temperature because, according to the Clausius-Clapeyron relation, saturation vapor pressure for any substance increases approximately exponentially with temperature. Although a variety of dynamical and microphysical effects prevent real atmospheres from attaining saturation, the actual condensible content nonetheless tends to scale with saturation vapor pressure (Pierrehumbert et al. 2007). The nondiluteness for a given temperature also depends on the mass of noncondensible background gas in the atmosphere, since a massive atmosphere can dilute a greater quantity of the condensible. The way these two factors play out for the case of condensible water vapor in noncondensible $\mathrm{N}_{2}$ is illustrated in Figure 1. In the nondilute case, one must take care with the way the quantity of noncondensible gas in the atmosphere is specified, as the noncondensible partial pressure becomes dependent on altitude and-for a fixed mass of the noncondensible-varies with temperature as the amount of condensible gas in the atmosphere changes. In this graph, we measure the amount of $\mathrm{N}_{2}$ in terms of the surface pressure the $\mathrm{N}_{2}$ would exert if it were present in the atmosphere alone, and we carry out the calculation in such a way that this quantity (and hence the mass of the noncondensible) remains fixed as temperature changes. We call this quantity the $\mathrm{N}_{2}$ inventory and refer to it by the symbol $p_{\mathrm{N}_{2}, I}$ (with analogous notation for other gases). For a given noncondensible inventory, the temperature at which the atmosphere becomes nondilute is independent of the planet's surface gravity. The corresponding mass of noncondensible gas A per square meter of the planet's surface, $p_{\mathrm{A}, I} / g$, is higher for planets with lower gravity, though.

The condensible fraction is greatest at the ground, so the atmosphere first becomes nondilute there. Hence, in Figure 1 we show the ground-level molar concentration of the condensible. When the $\mathrm{N}_{2}$ inventory is 0.01 bars, the low-level atmosphere is already roughly half water at temperatures of 


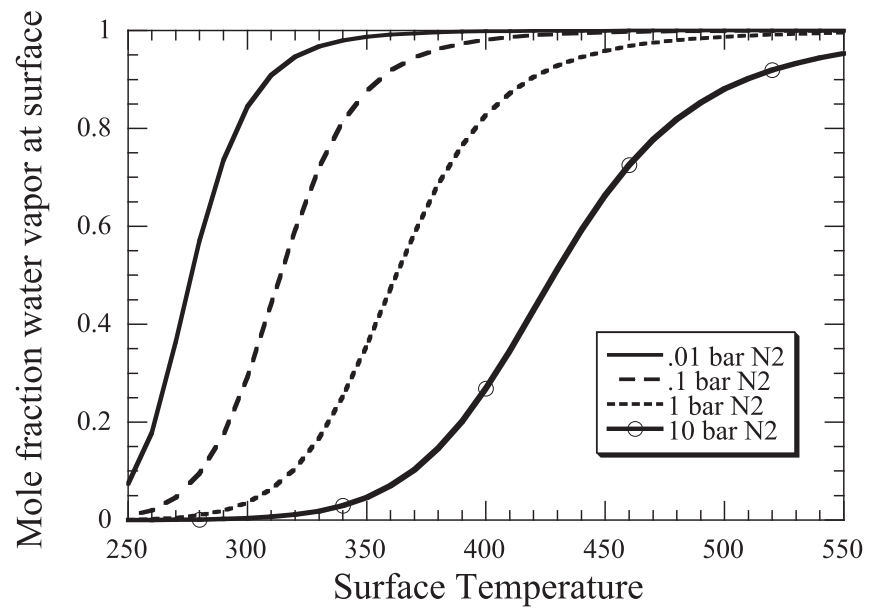

Figure 1. Molar concentration of water vapor at the surface as a function of the surface temperature. The calculation assumes that the atmosphere is fully saturated following the moist adiabat. The calculation is done for an atmosphere consisting of water vapor and noncondensible $\mathrm{N}_{2}$, holding the total atmospheric mass of $\mathrm{N}_{2}$ fixed as surface temperature is changed. The amount of $\mathrm{N}_{2}$ in the atmosphere for each curve shown is given in terms of the surface pressure the $\mathrm{N}_{2}$ would have if it were present in the atmosphere alone (i.e., $g \cdot M N_{2}$, where $g$ is the acceleration of gravity and $M\left(N_{2}\right)$ is the mass of $\mathrm{N}_{2}$ per square meter of surface).

$270 \mathrm{~K}$, whereas with a 1 bar $\mathrm{N}_{2}$ inventory (somewhat greater than the present Earth's) this concentration is not attained until the surface temperature exceeds $350 \mathrm{~K}$. With a $10 \mathrm{bar}$ inventory, the atmosphere is still dilute at $350 \mathrm{~K}$ and does not become strongly nondilute until $425 \mathrm{~K}$. Thus, the noncondensible inventory of an atmosphere is a crucial factor in determining the character of moist convection. This result underscores the importance of the factors governing the noncondensible inventory on a planet. Wordsworth \& Pierrehumbert (2013) found that a highly noncondensible inventory can also inhibit loss of water, and the reasoning in that paper applies to other condensible substances as well.

Temperature decreases with height on the adiabat, approaching zero as the pressure approaches zero, so within the convective region of an atmosphere-the troposphere-the atmosphere will become more dilute with height even if it is highly nondilute near the surface. Even in the radiativeequilibrium portion of the atmosphere-loosely speaking, the stratosphere-in the absence of atmospheric absorption of incoming stellar radiation, temperature decreases with altitude where the atmosphere is optically thick in some portion of the infrared spectrum. However, the temperature decrease ceases where the atmosphere becomes optically thin in the infrared, and suitably strong absorption of incoming stellar radiation can cause the temperature to increase with height, as in Earth's stratosphere (Pierrehumbert 2011, Chap. 4). Either effect can keep a nondilute lower atmosphere from being capped by a dilute upper troposphere or dilute stratosphere. The factors governing the diluteness of the stratosphere were discussed in detail in Wordsworth \& Pierrehumbert (2013), in connection with loss of volatiles to space.

All of the issues discussed in this paper are independent of the choice of condensible and noncondensible gas, though the choice of gases does affect the surface temperature at which the atmosphere begins to become nondilute, for any given inventory of noncondensible gas. For example, given a 1 bar inventory of noncondensible gas, $\mathrm{CO}_{2}$ condensing in an $\mathrm{N}_{2}$ atmosphere reaches $50 \%$ molar concentration at the ground for a surface temperature of $195 \mathrm{~K}$, and $\mathrm{CH}_{4}$ condensing in $\mathrm{N}_{2}$ reaches that molar concentration at $110 \mathrm{~K}$. For $\mathrm{N}_{2}$ condensing in an $\mathrm{H}_{2}$ background, the threshold is $95 \mathrm{~K}$. Here we have taken a molar concentration of $50 \%$ as an arbitrary boundary beyond which the nondilute effect is expected to be important. For some effects, notably the effect of the condensible content on the surface pressure, it is the mass fraction that is most directly relevant. The mass fraction differs greatly from the molar concentration for species with very different molecular weight. For $\mathrm{N}_{2}$ condensing in a 1 bar $\mathrm{H}_{2}$ background, the mass fraction is $93.3 \%$ at $50 \%$ molar concentration, so that $50 \%$ mass concentration would be attained at a much lower temperature than $95 \mathrm{~K}$.

The most familiar case in which nondilute physics becomes important is in the water vapor runaway greenhouse. This phenomenon has been extensively studied in one-dimensional radiative-convective models, but there is an increasing need to explore three-dimensional (3D) simulations of this phenomenon. Phenomena related to clouds, subsaturation, and geographical temperature and constituent variations can only be adequately treated in the context of a 3D GCM. Ishiwatari et al. (2002) carried out pioneering 3D GCM studies of the runaway greenhouse using gray-gas radiation and found that subsaturation played an important role in determining the runaway threshold. More recently, Leconte et al. (2013) and Yang et al. (2014) carried out simulations incorporating clouds and real-gas radiation, finding that clouds as well as subsaturation played a critical role. Wolf \& Toon (2015) carried out 3D simulations of a hot, moist early Earth atmosphere up to temperatures of $360 \mathrm{~K}$, which brings the lower troposphere well into the nondilute regime; convection in this case is treated through a modification of the ZhangMacfarlane convection scheme, but the nature of the modifications was not discussed. None of these studies explicitly discussed the novel aspects of convection in the nondilute case, or the physics involved in maintaining energy conservation. Generally speaking, there is a need for convection parameterizations that cover the nondilute limit accurately and are suitable for incorporation in GCMs. There is also a broad need for a better understanding of how moist convection operates in nondilute systems.

There are many other cases in which nondilute physics is important. Methane is a nondilute condensible on Titan, making up about $30 \%$ of its lower atmosphere when saturated. $\mathrm{CO}_{2}$ is nondilute on early Mars, and more generally near the outer edge of the conventional habitable zone where habitability is supported by the greenhouse effect of a thick $\mathrm{CO}_{2}$ atmosphere whose mass is limited by $\mathrm{CO}_{2}$ condensation onto the surface (Wordsworth et al. 2011). As is evident from Figure 1, a low noncondensible inventory can cause water vapor to be nondilute even at temperatures comparable to the present Earth; as such atmospheres are prone to losing water to space and generating abiotic oxygen (Wordsworth \& Pierrehumbert 2014), the dynamical features that could affect water loss are of considerable interest.

The dilute approximation enters into the formulation of convection parameterizations used in conventional GCMs in several ways. First, it is used in the approximate form of moist enthalpy to enforce energy conservation in physical processes involving condensation. Second, it is invoked in order to neglect the energy carried away by precipitation. Third, it is 
used to justify the neglect of pressure changes that occur when condensate mass is added to the atmosphere by evaporation or taken away by precipitation. When the convection is nondilute, the pressure effect can be important in driving atmospheric circulations. Moreover, removing mass from a layer of the atmosphere by precipitation unburdens the layers below, causing them to expand and do pressure work. Finally, in the nondilute case there is a need to carefully take into account the effect of changing atmospheric composition on mean thermodynamic quantities such as specific heats or gas constants (though some existing parameterizations already take such things into account, at least approximately).

Many of the novel aspects of nondilute convection affect energy conservation properties. In situations where it is necessary to accurately track transient behavior (e.g., in seasonal or diurnal cycles) or in which energy is transported from one geographical location to another (as in a GCM), energy conservation becomes crucial. Even in a steady state in a single-column model, energy conservation is important in models that explicitly resolve the surface energy balance, since energy deposited at the surface is transferred to the lowest model layer, after which it needs to be communicated upward by convection without loss or gain. The only situation in which energy conservation in the course of convective adjustment is unimportant is that in which detailed modeling of energy transfer between surface and atmosphere is replaced by an assumption that the surface temperature equals the immediately overlying air temperature, provided that only the steady state with infrared radiation to space balancing net absorbed stellar radiation is of interest. This is a fairly common approach in radiative-convective models, which has allowed many studies (e.g., Kasting 1991) to avoid the necessity of confronting energy conservation in nondilute convection.

In this paper, we formulate and test a simplified convection parameterization scheme that can deal with the full range of situations from dilute to condensible-dominated behavior, and we use the scheme to explore some of the novel aspects of nondilute moist convection. The scheme is suitable for use both in single-column radiative-convective models and in GCMs. The scheme applies to any combination of a single condensible substance and noncondensible background gas and conserves energy for any concentration of the condensible component.

The development of our scheme builds in part on the treatment of convection in a pure $\mathrm{CO}_{2}$ Martian atmosphere given in Forget et al. (1998) and the related scheme for thicker atmospheres employed in Wordsworth et al. (2011). Leconte et al. (2013) incorporated some aspects of nondilute behavior in their 3D runaway greenhouse study but did not specifically discuss the convection scheme, its energy conservation properties, or the nature of nondilute convection. Further development of the subject requires a broader discussion of the basic ways in which nondilute convection differs from the familiar dilute case.

In Section 2 we present a complete description of the convection scheme. We show that this scheme conserves energy whether the condensible substance is dilute in the atmosphere or not. As an example of the behavior of the scheme, we apply it to an atmosphere undergoing a runaway greenhouse on a planet with a high eccentricity orbit in Section 3. This serves as a test of energy conservation in a situation involving strong transient forcing. We discuss key differences between dilute and nondilute convection, as revealed by our simulations, in Section 4, and we summarize our main findings, together with pointers toward unresolved issues, in Section 5.

\section{DESCRIPTION OF THE MOIST CONVECTION SCHEME}

Manabe \& Strickler (1964) first introduced a convective adjustment scheme in their study of a single air column subject to atmospheric radiation and convection. In their convection scheme, once the vertical lapse rate in the model exceeds a critical value (e.g., $6.5 \mathrm{~K} \mathrm{~km}^{-1}$, a typical value for present Earth's midlatitude atmosphere), it is reset to the critical value. This assumes that the free convection is strong enough to maintain the assumed critical lapse rate. The critical lapse rate determines only the local slope of the adjusted $T(p)$, and an additional principle must be invoked to determine the intercept. In the initial version of the Manabe scheme, the columnintegrated dry enthalpy $\int_{0}^{p_{s}}\left(c_{p} T\right) d p / g$ is assumed to be conserved during the convective adjustment, where $p_{s}$ is the surface pressure, $c_{p}$ is the specific heat of dry air, and $g$ is the acceleration of gravity. Energy stored in the form of latent heat was not taken into account. Manabe et al. (1965) modified this convection scheme for a moist atmosphere when studying the climatology of a GCM with a simple hydrological cycle. This parameterization used the moist adiabat as the critical lapse rate and conserved the column-integrated moist enthalpy in the dilute limit $\int_{0}^{p_{s}}\left(c_{p} T+L q\right) d p / g$ during the convective adjustment, where $L$ is the latent heat of condensation and $q$ is the mass mixing ratio of water vapor. Betts (1986) and Betts \& Miller (1986) proposed another new convection scheme (now known as the Betts-Miller scheme) by relaxing the temperature and humidity profiles gradually toward the post-convective equilibrium states with a given relaxation timescale. The precipitation rate and the change of the temperature and humidity profiles during the convective adjustment are again computed using conservation of the column-integrated moist enthalpy in the dilute limit. These schemes are widely used in idealized experiments (Rennó et al. 1994; Frierson 2007; O'Gorman \& Schneider 2008; Merlis \& Schneider 2010).

In this paper, we propose a new simplified convection scheme similar in spirit to the convective adjustment scheme in Manabe \& Strickler (1964) and the Betts-Miller scheme, but without assuming the condensible substance to be dilute. The resulting scheme conserves nondilute moist enthalpy, takes the enthalpy of condensate and the mass loss from precipitation into account, and works for both dilute and nondilute cases no matter what the condensible substance is.

The atmosphere is divided into discrete layers, and the scheme is applied iteratively to adjacent pairs of layers. The adjustment is performed for each pair of layers from bottom to top, with the procedure being repeated until the temperature and moisture profiles converge to their asymptotic form within the specifed degree of accuracy.

When a pair of adjacent layers is found to be unstable to convection, adjustment to a neutral state is performed through a two-step process. The subdivision of the adjustment into steps makes it easier to assure that each step (and hence the whole process) conserves energy. First, the pair is adjusted to the reference neutrally stable state of temperature and humidity conserving the summed nondilute moist enthalpy and moisture of the two layers, assuming that the final state is saturated in both layers. Condensate formed in this step is retained in each 
layer in such a way that the total mass of each layer remains unchanged; hence, in this step the pressure of the interface between the layers and of lower layers remains unchanged, in accordance with hydrostatic equilibrium. If there is not enough moisture present to saturate both layers, the adjustment is done without forming any condensate, in a manner that will be detailed later.

In the second step, condensate is removed from the layers. For simplicity, we currently assume infinite precipitation efficiency, so that all precipitation is transported to the surface without any evaporation along the way; we also neglect heating due to frictional dissipation associated with the falling precipitation. The scheme can be easily modified to incorporate these complicating effects, but here we wish to focus on the most basic effects of nondiluteness. When condensate is removed, the atmosphere is allowed to adjust to the resulting change in pressure, and the energy carried by precipitation is tracked, in such a way that the system conserves energy in the course of precipitation.

When mass is removed from the atmosphere by precipitation, the potential and thermal energy of the precipitation must be added to the surface energy reservoir for energetic consistency. At the bottom boundary, surface fluxes remove energy and condensate mass from the surface reservoir and add it to the lowest model layer. In Sections 2.3 and 2.4 we show how the energy budget is balanced in the course of these processes.

\subsection{Thermodynamic Preliminaries: Nondilute Moist Enthalpy and the Nondilute Pseudoadiabat}

We begin with a review of some relations pertinent to energy conservation in the nondilute case. The calculations involve three atmospheric constituents: a noncondensible gas (or mixture of gases) referred to with subscript $a$, and a condensible substance whose gaseous form is referred to by subscript $c$ and whose condensed form is referred to by subscript $\ell$. The subscript $t$ will be used to refer to quantities associated with the total mass of the condensible in both phases.

The temperature dependence of latent heat enters the problem at several points. When the density of the condensate is much greater than that of the vapor phase, the specific latent heat varies linearly with the temperature (Emanuel 1994, p. 115):

$$
\frac{d L}{d T} \approx c_{p c}-c_{p \ell}
$$

Here subscript $\ell$ represents the condensate, which may be in a solid or liquid phase. We will take this temperature dependence into account in our formulation, but we assume the specific heats to be independent of temperature.

Next, consider a parcel of atmosphere that has become supersaturated by one means or another and that relaxes back to saturation through condensation without loss of energy or mass. Thus,

$$
d m_{c}+d m_{\ell}=0, \quad d m_{c}<0,
$$

where $m_{c}$ and $m_{\ell}$ are the mass of condensible gas and condensate in the parcel, respectively. Suppose further that the pressure is kept constant in the course of the condensation. In hydrostatic equilibrium, this would correspond to the situation in which no mass is lost from the column of atmosphere above the parcel in question. The first law of thermodynamics then implies

$$
0=L(T) d m_{c}+\left(c_{p a} m_{a}+c_{p c} m_{c}+c_{p \ell} m_{\ell}\right) d T
$$

where $m_{a}$ is the mass of the noncondensible in the parcel. From Equation (1) and the assumption that specific heats do not depend on temperature, we deduce

$$
L(T) d m_{c}=d\left[L(T) m_{c}\right]-\left(c_{p c}-c_{p \ell}\right) m_{c} d T .
$$

Combining this result with Equation (3), we can define a quantity that is conserved in the course of isobaric condensation without loss of mass or energy:

$$
\tilde{k}=\left(c_{p a} m_{a}+c_{p \ell} m_{t}\right) T+L(T) m_{c},
$$

where $m_{t}=m_{c}+m_{l}$. The conserved variable $\tilde{k}$ is usually referred to as the moist enthalpy (Emanuel 1994, p. 118). Dividing by $m_{a}+m_{t}$ yields the moist enthalpy per unit total mass

$$
k=\left(c_{p a} q_{a}+c_{p \ell} q_{t}\right) T+L(T) q_{c},
$$

where $q$ is the mass concentration of the atmospheric constituent indicated by each subscript. Allowing for pressure work done by the parcel in the general case, the first law becomes

$$
d k-\frac{1}{\rho} d p=\delta Q
$$

where $\rho$ is the total density of all constituents and $\delta Q$ is the energy change due to such processes as radiative heating or cooling. Alternately, in a temperature-volume formulation the first law can be written as

$$
d\left(k-\frac{p}{\rho}\right)+p d \frac{1}{\rho}=\delta Q .
$$

Equations (7) and (8) are conservation laws that apply following an individual air parcel, but they are not generally the same as the conservation law that applies when a column consisting of various air parcels is mixed by convection. Even if the initial and final states of the column are in hydrostatic balance, convection proceeds through nonhydrostatic motions, so energy conservation must be formulated to allow for nonhydrostatic dynamics. This is most easily done using altitude $(z)$ rather than pressure $(p)$ as the vertical coordinate. In the Appendix it is shown that an isolated volume of atmosphere conserves the quantity

$$
\int_{z=0}^{\infty} \rho \cdot\left(k-\frac{p}{\rho}+g z\right) d z
$$

upon mixing by fluid motions of an arbitrary type, provided that any condensate formed is retained within the air parcel in which it forms (though it is free to evaporate back into the air parcel). The density $\rho$ in this formula includes the mass of the condensate. This proceeds from a small variant of a standard thermodynamic derivation, but it is reproduced in the Appendix for the sake of checking that it remains valid even in the nondilute limit, and even in the presence of the condensate. The conservation law given in Equation (9) is an approximate form of the exact conservation law, valid when kinetic energy 
in the initial and final states is negligible compared to the thermal energy. The energy contains contributions from both the internal energy $k-p / \rho$ and potential energy $g z$. In the dilute case for an ideal gas, $q_{a} \approx 1, p / \rho \approx R_{a} T, q_{t} \ll q_{a}$, and the internal energy takes on the familiar form $c_{v a} T+L q_{c}$. Returning to the general case, if the column is in hydrostatic balance, then $g \rho d z=-d p$ and

$$
\int_{z=0}^{\infty} \rho g z d z=\int_{p=0}^{p_{s}} z d p=-\int_{p=0}^{p_{s}} p d z=\int_{z=0}^{\infty} p d z
$$

where $p_{s}$ is the surface pressure. Thus, for a column in hydrostatic balance the potential energy term cancels the term in Equation (9) arising from $p / \rho$, whence we conclude that the quantity

$$
\int_{p=0}^{p_{s}} k d p
$$

is conserved in the course of mixing of a column of atmosphere provided that the initial and final states are in hydrostatic balance and no mass is lost from any air parcel in the course of the mixing. The potential energy does not appear explicitly in this expression, but it is implicitly accounted for through the fact that $c_{p}$ rather than $c_{v}$ appears in the expression for enthalpy $k$. This will be important when we come to consider the energy carried by precipitation that removes mass from the atmosphere. One knows intuitively that part of the energy carried by the precipitation should be in the form of potential energythat is after all where hydroelectric power comes from, namely, the potential energy of water vapor stored in the atmosphere when solar energy is used to drive convection, which lifts the water vapor to higher altitudes. Without carefully considering the above derivation, it is not clear where this potential energy is taken from when mass is removed from the atmosphere. The energy bookkeeping is transparent if done in $z$ coordinates but is more subtle when done in $p$ coordinates for a hydrostatic atmosphere. We will return to this point in Section 2.3, where we deal with mass loss.

We use the pseudoadiabat (Pierrehumbert 2011) as the reference temperature profile, meaning that the condensate does not accumulate in the atmosphere to the extent that the temperature profile would be significantly affected. By substituting the expression for $d \ln p_{a} / d \ln p$ into the formula given in Pierrehumbert (2011), the pseudoadiabatic slope can be written in the form

$$
\begin{aligned}
\frac{d \ln p}{d \ln , T}= & \frac{p_{\mathrm{sat}}}{p} \frac{L(T)}{R_{c} T}+\frac{p_{a}}{p} \frac{c_{p a}}{R_{a}} \\
& \times \frac{1+\left(\frac{c_{p c}}{c_{p a}}+\left(\frac{L}{R_{c} T}-1\right) \frac{L}{c_{p a} T}\right) r_{\mathrm{sat}}}{1+\frac{L}{R_{a} T} r_{\mathrm{sat}}} .
\end{aligned}
$$

This expression remains valid even in the nondilute case. Here $p_{\text {sat }}$ is the saturation vapor pressure, $L$ is the specific latent heat of vaporization, $R$ is the specific gas constant, $c_{p}$ is the specific heat capacity at constant pressure, and $r_{\mathrm{sat}}$ is the saturation mass mixing ratio. Note that the pseudoadiabat reduces to the Clausius-Clapeyron relation at temperatures high enough that $r_{\text {sat }} \gg 1$, in which case $p \rightarrow p_{\text {sat }}$ and $p_{a} / p \rightarrow 0$.

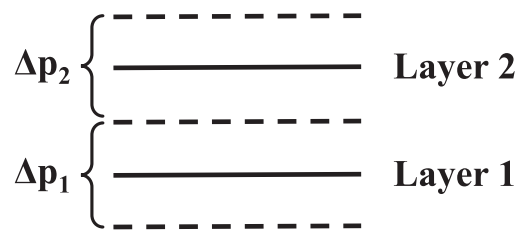

Figure 2. Notations used for the moist convective adjustment.

\subsection{Lapse Rate Adjustment with Retained Condensate}

Consider two adjacent layers of atmosphere arranged as in Figure 2. The lower layer has pressure and temperature $\left(p_{1}, T_{1}\right)$ and pressure thickness $\Delta p_{1}$, while the upper layer has pressure and temperature $\left(p_{2}, T_{2}\right)$ and pressure thickness $\Delta p_{2}$.

In the first step of the convection scheme, we check the temperature difference between the two layers. If it is steeper than the pseudoadiabat computed on the basis of Equation (12), heat and moisture are assumed to mix between the two layers, resulting in a state that satisfies the following criteria:

1. The temperature difference between the layers is given by the slope of the pseudoadiabat.

2. The vapor phase of the condensible is saturated in each layer, provided that the mass of the condensible in the adjusted state is less than or equal to the available condensible mass in the initial state.

3. The excess of condensible mass between the initial and final state is turned into condensate, which is retained within the two layers.

4. The net nondilute moist enthalpy of the two layers is the same as in the initial state.

5. The net mass in each layer remains unchanged, so according to the hydrostatic relation, $\Delta p_{1}$ and $\Delta p_{2}$ are the same in the initial and adjusted states.

The last of these assumptions is not really a physical assumption, but rather a convention about how the pressure layer interfaces are labeled after mixing takes place. This set of requirements gives rise to a nonlinear relation with one unknown parameter, which can be taken to be the temperature of the lower layer after adjustment; this parameter is adjusted using a Newton iteration until the conditions are satisfied to within a specified accuracy.

Specifically let $T_{1, I}$ be the lower layer temperature for the initial state and $T_{1, F}$ be the lower layer temperature for the adjusted state, with analogous notation for the other quantities. The temperature difference $\Delta T \equiv T_{1}-T_{2}$ on the pseudoadiabat is computed from Equation (12), and if $T_{1, I}-T_{2, I}>\Delta T$, convection is initiated and the temperatures are reset to

$$
T_{1}=T_{1, F}, T_{2}=T_{1, F}-\Delta T
$$

where $T_{1, F}$ is yet to be determined. Because condensate forms in the course of the adjustment, it is most convenient to express the saturation assumption in terms of the mixing ratios $r_{c}$ of the gas-phase condensible relative to the noncondensible component:

$$
r_{c 1, F}=r_{\mathrm{sat}}\left(T_{1, F}, p_{1}\right), r_{c 2, F}=r_{\mathrm{sat}}\left(T_{2, F}, p_{2}\right)
$$

where $r_{\mathrm{sat}}(T, p)$ is the function giving the mixing ratio at saturation. Conservation of mass of the condensible substance 
between the initial and adjusted states requires

$$
\begin{gathered}
\frac{r_{c 1, F}+r_{\ell 1, F}}{1+r_{c 1, F}+r_{\ell 1, F}} \Delta p_{1}+\frac{r_{c 2, F}+r_{\ell 2, F}}{1+r_{c 2, F}+r_{\ell 2, F}} \Delta p_{2} \\
=q_{c 1, I} \Delta p_{1}+q_{c 2, I} \Delta p_{2}
\end{gathered}
$$

since there is assumed to be no retained condensate in the initial state. This yields only one constraint for the two unknowns $r_{\ell 1, F}$ and $r_{\ell 2, F}$. Because total mass is conserved in each layer, the expression for conservation of noncondensible mass does not yield an independent relation. In order to close the problem, an assumption must be made regarding the distribution of condensate between the two layers. Defining $\eta \equiv r_{\ell 2, F} / r_{\ell 1, F}$ allows Equation (15) to be solved for $r_{\ell 1}$, whence multiplication by $\eta$ gives $r_{\ell 2}$. The left-hand side of Equation (15) is monotonic in $r_{\ell 1}$ and approaches $\Delta p_{1}+\Delta p_{2}$ for $r_{\ell 1} \rightarrow \infty$, which is guaranteed to be greater than the righthand side because $q_{c} \leqslant 1$. If the initial state has enough condensible to saturate both layers, then the left-hand side is less than the right-hand side when $r_{\ell 1}=r_{\ell 2}=0$, whence we conclude that there is a unique solution with positive $r_{\ell}$. If this condition is not satisfied, there is no physical solution. We will discuss the handling of that case shortly.

With the above assumptions, it is possible to compute the enthalpies $k_{1, F}$ and $k_{2, F}$ in the adjusted layers given $T_{1, F}$. Then $T_{1, F}$ is adjusted using a Newton iteration until the enthalpy conservation relation

$$
k_{1, F} \Delta p_{1}+k_{2, F} \Delta p_{2}=k_{1, I} \Delta p_{1}+k_{2, I} \Delta p_{2}
$$

is satisfied.

There is no clear physical basis for determining $\eta$, but we do not believe that it is a critical parameter of the scheme, since all condensate is removed in the second stage of the adjustment process. In the calculations reported in this paper we adopted the choice

$$
\eta=\frac{1+r_{c 2}}{1+r_{c 1}}
$$

which implies that condensate mass is distributed in proportion to the gas-phase mass of the atmosphere. This was found to yield a stable iteration and also has the virtue of allowing Equation (15) to be solved analytically for $r_{\ell 1}$ in terms of a simple linear equation.

It sometimes happens that there is not enough moisture in the initial state to allow a saturated adjusted state to be realized, i.e., that the adjusted saturated state would require negative precipitation. In such a case we still adjust the temperature to the pseudoadiabat in an enthalpy-conserving way, but we do not form any precipitation. In this case, the condensible vapor is redistributed in proportion to the final-state saturation mixing ratio, with a proportionality constant $f_{1}<1$ chosen to conserve the condensible mass between the initial and final state:

$$
r_{c 1, F}=f_{1} r_{\text {sat }}\left(T_{1, F}, p_{1}\right), r_{c 2, F}=f_{1} r_{\text {sat }}\left(T_{2, F}, p_{2}\right) .
$$

The temperature and moisture adjustment employed here are similar to the treatment of shallow nonprecipitating convection in the simplified Betts-Miller scheme (Frierson 2007) used in many conventional GCM studies.

Condensation can also happen in the absence of convection, and such condensation is generally referred to as large-scale condensation. In a 3D GCM it can happen as a result of uplift and adiabatic cooling caused by the resolved large-scale circulation, but it can also be caused by radiative cooling; the latter mechanism is the only large-scale condensation mechanism in a 1D radiative-convective model. When large-scale condensation forms, it can simply be added to the condensate (if any) produced by the convection step and dealt with in the precipitation scheme described in the next section.

\subsection{Precipitation and Mass Loss from the Atmosphere}

In the precipitation step, condensate mass is removed from the atmosphere one layer at a time. The precipitation is assumed to reach the surface instantaneously without loss of energy or mass along the way. When this happens, an amount of enthalpy $c_{p, \ell} T q_{\ell} \Delta p / g$ is removed from the layer and added to the surface energy budget. The temperature of the remaining gas in the layer remains unchanged, but the layer thickness $\Delta p$ goes down in accord with the mass loss from the layer. This reduces the pressure of all the layers located at lower altitudes. When condensate is removed, it is like taking a brick off the top of a piston supporting a column of air; the piston rises, and the air in the cylinder below the piston expands adiabatically until the reduction in pressure force equals the new force of gravity exerted by the mass of the piston. In the course of the expansion, pressure work is done.

In the context of an atmospheric column, the enthalpy of layers below the one from which condensate was removed goes down to compensate for the pressure work done. This reduction is manifest as a reduction in the temperature of the lower layers, but because no mass is taken away from the lower layers in this step, their individual pressure thicknesses remain unchanged. The temperature reduction typically leads to supersaturation, but that is dealt with at the next condensation and convection step.

The pressure work done in the course of the expansion of the lower layers, and hence the enthalpy reduction in those layers, can be computed as follows. Let $p_{1}$ be the pressure of the level from which a condensate mass (per unit area) $\delta m$ was removed, and let $z_{1}$ be the corresponding altitude. Then the work per unit mass done at some level $p<p_{1}$ is

$$
\frac{1}{\rho} \delta p=\frac{1}{\rho} g \delta m .
$$

Then, doing a mass-weighted integral of this over all the lower layers yields the pressure work

$$
\begin{aligned}
\delta W & \equiv \int_{p_{1}}^{p_{s}}\left[\frac{1}{\rho} \delta p\right] \frac{1}{g} d p=g \delta m \int_{p_{1}}^{p_{s}} \frac{1}{\rho} \frac{1}{g} \frac{d p}{d z} d z \\
& =g \delta m \int_{0}^{z_{1}} d z=g z_{1} \delta m .
\end{aligned}
$$

This is precisely the potential energy of the precipitation removed. Hence, energy conservation in the column is achieved if the potential energy, as well as the enthalpy, of the precipitation is added to the surface budget. In our simplified model, the potential energy is implicitly converted to kinetic energy of falling precipitation, which is then dissipated as heat when the precipitation strikes the surface. Forget et al. (1998) also included the potential energy of precipitation in the convection scheme used in their study of 
$\mathrm{CO}_{2}$ snowfall on Mars, but they did not explicitly consider the way the inclusion of this term leads to energy conservation.

\subsection{Treatment of Evaporation}

Evaporation is most easily treated in terms of discrete layers, rather than trying to pass to the continuum limit. Evaporation from the surface reservoir of the condensible adds condensible mass to the lowest layer of the atmosphere, increasing its gasphase condensible content and also increasing its mass and hence the pressure thickness $\Delta p$ of the layer. The pressure of higher layers is not affected, though implicitly potential energy is being stored in higher layers since, viewed in $z$ coordinates, the addition of mass to the lowest layer raises the altitude of all overlying layers, insofar as the added mass takes up space. Additional energy is stored in the lowest model layer in the form of the latent heat of the vapor added to the layer, which is equal to the energy lost from the planet's surface through evaporation.

Since the temperature dependence of the latent heat is taken into account, it may seem that the latent heat change during atmospheric condensation aloft is larger than the latent heat put into the atmosphere during surface evaporation of the same amount of mass. However, the thermal energy change on converting a mass $\delta m_{\ell}$ from vapor to liquid at temperature $T$ is

$$
\left[L(T)+\left(c_{p l}-c_{p c}\right) T(p)\right] \delta m_{\ell} .
$$

This reflects the loss of sensible heat from the vapor phase and the gain in the form of sensible heat of the condensate, as well as the latent heat of phase change. From Equation (1) for the temperature dependence of the latent heat, the term in brackets is simply $L(0)$, which is independent of temperature. Hence, the net energy change from condensation or evaporation is independent of temperature; the difference between latent heat added near the surface and latent heat released aloft is accounted for in the sensible heat carried away by the precipitation.

\section{RESULTS}

We carry out three experiments using a 1D column model incorporating the convection scheme introduced in Section 2. The first two simulations verify that the convection scheme conserves energy and mass for both dilute and nondilute cases. In the third simulation we apply the model to the atmosphere undergoing the runaway greenhouse on a planet with a higheccentricity orbit and illustrate some additional features of convection in the nondilute limit.

\subsection{Model Framework}

The noncondensible substance in the model is the mixed $\mathrm{N}_{2}-$ $\mathrm{O}_{2}$ air on Earth, and the mass of the noncondensible substance, unless otherwise noted, is $10^{4} \mathrm{~kg} \mathrm{~m}^{-2}$, as in the present Earth's atmosphere. The condensible substance in the model is water. The moist convection scheme determines the level of the tropopause. We then calculate the level of the cold trap where the saturation concentration of water vapor reaches its minimum above the tropopause and assume uniform concentration above the cold trap and relative humidity of unity between the tropopause and the cold trap. This is equivalent to a simplified vertical diffusion scheme. The calculation is performed with a gravitational acceleration of $9.8 \mathrm{~m} \mathrm{~s}^{-2}$.
The column model uses a gray radiation scheme similar to that described in Merlis \& Schneider (2010) in the longwave spectral region. As in Merlis \& Schneider (2010), the absorption coefficient of water vapor is kept constant at $0.1 \mathrm{~m}^{2} \mathrm{~kg}^{-1}$, but unlike Merlis \& Schneider (2010), we compute the water vapor optical thickness based on the actual water vapor path in the model, rather than an idealized profile. With the stated absorption coefficient, the effective radiating level is $0.98 \mathrm{hPa}$ for pure water vapor atmosphere. Therefore, in order to resolve the radiative and convective process at high water concentrations, we choose the level of $0.01 \mathrm{hPa}$ as the top of the column model. In addition to the water vapor opacity, which varies with the water content (and hence temperature) of the atmosphere, the model includes a fixed background opacity that on its own would give the atmosphere a longwave optical thickness $\tau_{0}=1.2$. This can be thought of as the opacity due to a noncondensible trace gas in the atmosphere, such as $\mathrm{CO}_{2}$. With these parameters the curve of the outgoing longwave radiation (OLR) as a function of the surface temperature exhibits overshoot for a saturated atmosphere, peaking at a value of $\mathrm{OLR}_{\max }=258 \mathrm{~W} \mathrm{~m}^{-2}$ at $T=320 \mathrm{~K}$ before asymptoting to a value $\mathrm{OLR}_{\infty}=210 \mathrm{~W} \mathrm{~m}^{-2}$ at high $T$ (see Figure 10). Thus, if the absorbed stellar radiation is below $210 \mathrm{~W} \mathrm{~m}^{-2}$, the system can never enter a runaway greenhouse, and if it is above $258 \mathrm{~W} \mathrm{~m}^{-2}$, the system runs away regardless of the initial condition. For absorbed stellar radiation between 210 and $258 \mathrm{~W} \mathrm{~m}^{-2}$ the system will run away if initialized warmer than $320 \mathrm{~K}$ but will be attracted to a metastable nonrunaway state if initialized cooler than $320 \mathrm{~K}$.

We assume that the atmosphere is transparent to the incoming stellar radiation. In reality the near-infrared absorption and Rayleigh scattering by water vapor molecules are very important when the water content in the atmosphere is high, but here we are chiefly interested in illustrating the behavior of the convection scheme, particularly with regard to vertical energy and moisture transport. The relevant phenomena are brought out most clearly in the idealized case in which incoming energy is deposited only at the surface.

The simulations shown are carried out with five iterations per time step and a time step of 40 minutes in the dilute and runaway simulations and 20 minutes in the equilibrium nondilute simulation.

The lower boundary of the column model is a heat reservoir with fixed thickness of $1 \mathrm{~m}$ and specific heat equal to that of liquid water. This lower boundary is included as a computational device to allow the surface energy budget to relax smoothly toward equilibrium; while it is mathematically identical to the common representation of an ocean mixed layer, it is not intended to represent either a shallow ocean or the mixed layer of an actual deep ocean. To represent an actual ocean layer, one would need to allow the depth to change in response to the mass budget, which is a simple modification but an unnecessary complication in view of the use to which the heat reservoir is put in our model. At the surface, the sensible and latent heat fluxes are computed by standard drag laws assuming constant drag coefficients and surface wind velocity. The surface is also heated or cooled by absorption of the shortwave radiation transmitted through the atmosphere and the net surface infrared flux (computed within the gray-gas model). The chief novel aspect of the surface budget in the nondilute case is that the enthalpy of the precipitation is added to the surface budget, as is the kinetic energy of the 


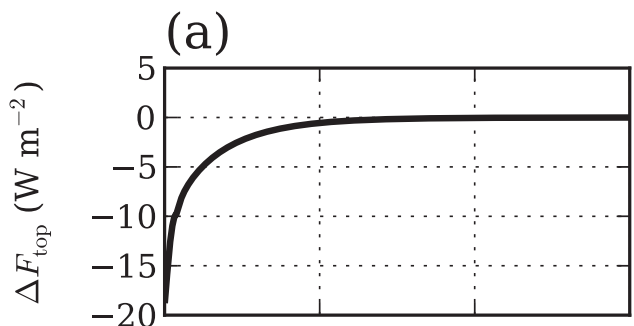

(b)

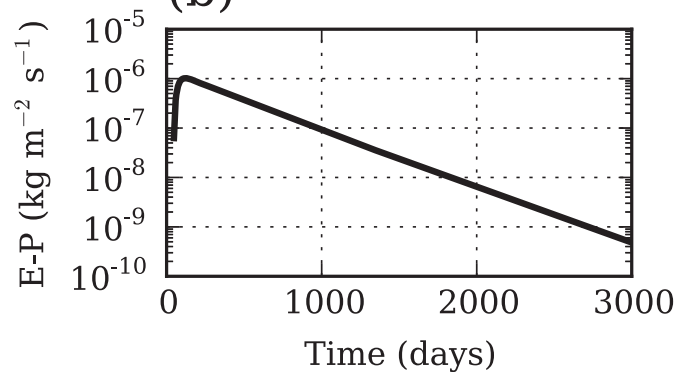

(c)

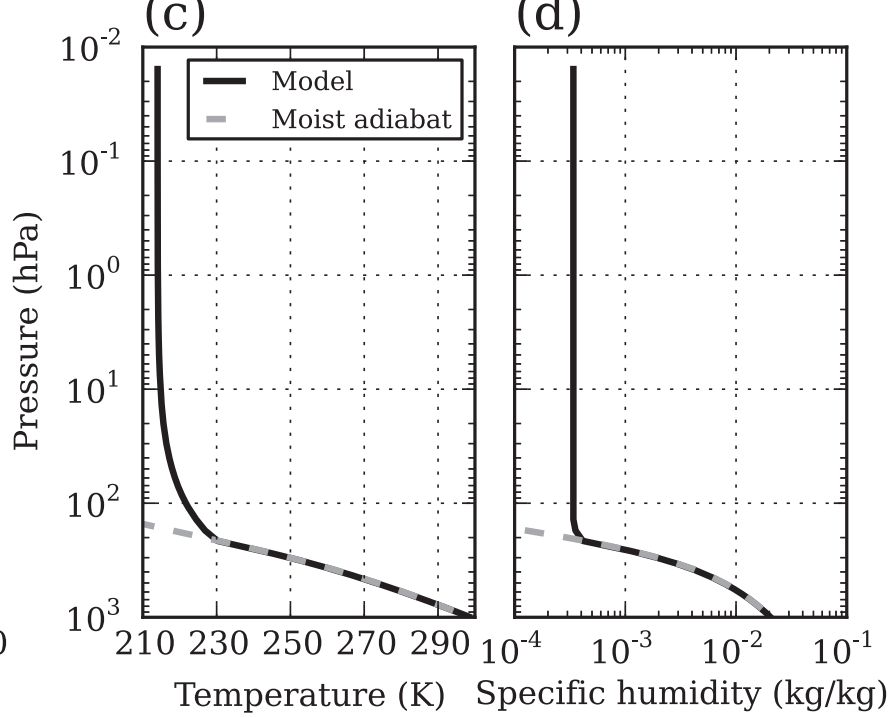

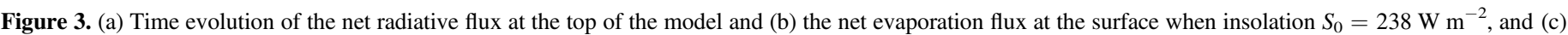

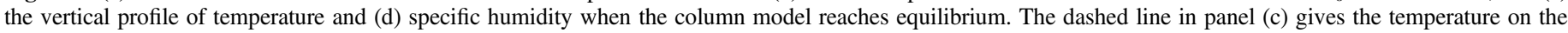
moist adiabat, and the dashed line in (d) gives the corresponding moisture in saturation.

(a)

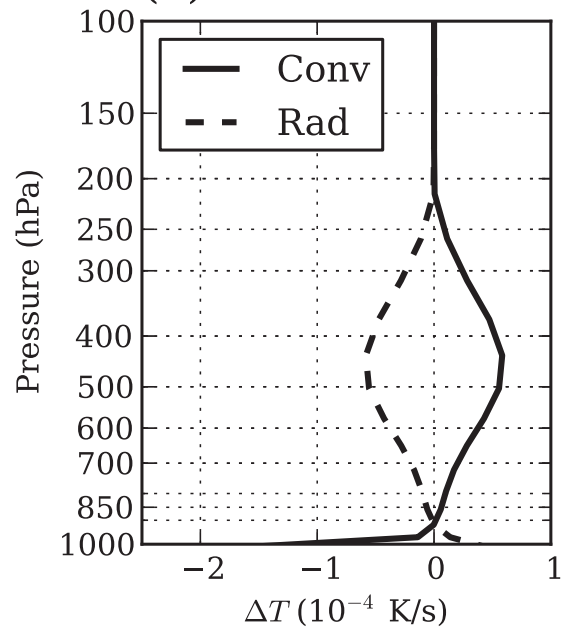

(b)

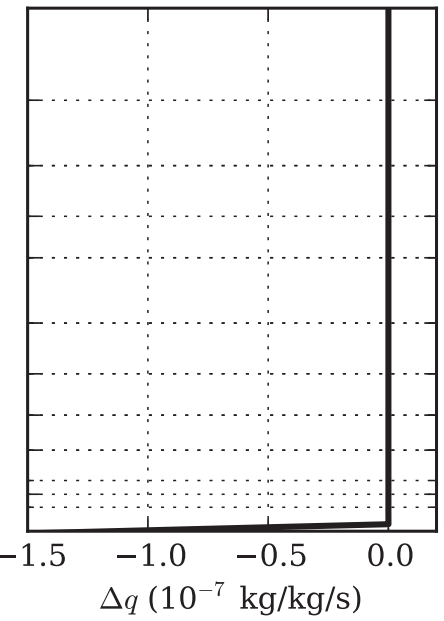

(c)

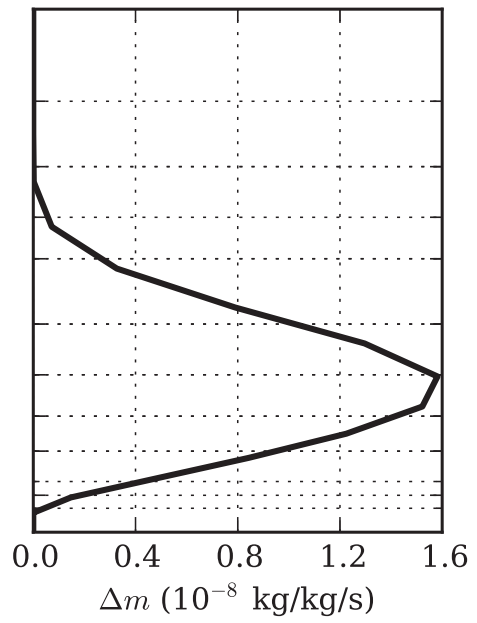

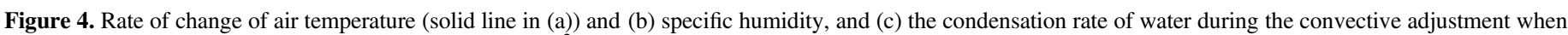

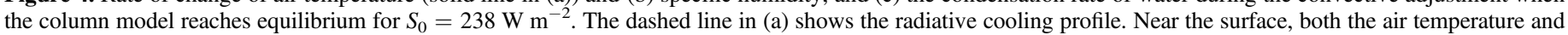
specific humidity decrease during the convective adjustment primarily owing to the upward transport of sensible heat and moisture.

precipitation (after being converted to heat). In addition, surface pressure is updated by the net evaporation rate at the surface at the end of each time step.

\subsection{Dilute Simulation}

In the dilute simulation, we use the average shortwave solar flux absorbed by Earth's climate system $S_{0}=238 \mathrm{~W} \mathrm{~m}^{-2}$ as the insolation and assume that the albedo of the surface is zero. With the assumed background noncondensible opacity the equilibrium surface temperature in this simulation is similar to Earth's present tropics, and the system is therefore in the dilute regime.

We first check whether the convection scheme conserves energy. Both the net radiative flux at the top of the model and the net evaporation flux at the surface nearly vanish after integration of 3000 days, implying that the system reaches not only energy but also mass equilibrium (Figures 3(a) and (b)).
The equilibrium profiles of air temperature and specific humidity are shown in Figures 3(c) and (d). Below $\sim 200 \mathrm{hPa}$ the atmosphere follows the moist adiabat, which defines the convective region. Above the tropopause, the atmosphere is in radiative equilibrium. Since there is no ozone in the column model, the upper atmosphere is nearly isothermal owing to the gray-gas assumption. The specific humidity at the surface is $\sim 2.08 \times 10^{-2} \mathrm{~kg} \mathrm{~kg}^{-1}$, confirming that water vapor is dilute in this simulation.

The behavior of the convection scheme is shown in Figure 4. The troposphere is heated by the latent heat release and the convergence of sensible heat flux, except near the surface. In equilibrium, this heating is balanced by the radiative cooling in the troposphere. Near the surface, most of the sensible heat is transported upward and little condensation occurs, resulting in a cooling effect that is balanced by heat input from the surface (which is in turn heated by solar absorption). The maximal 
(a)

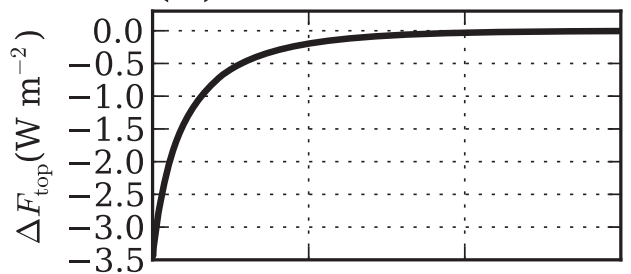

(b)

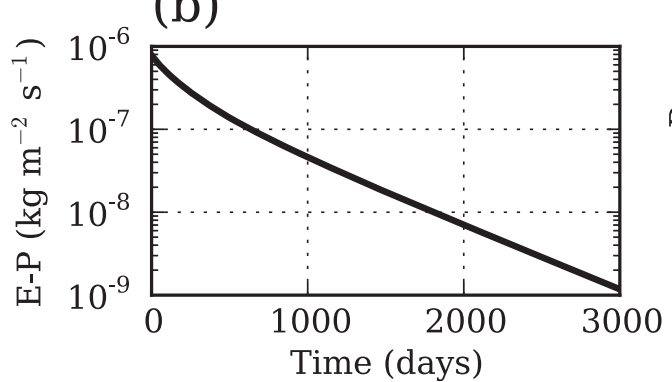

(c)

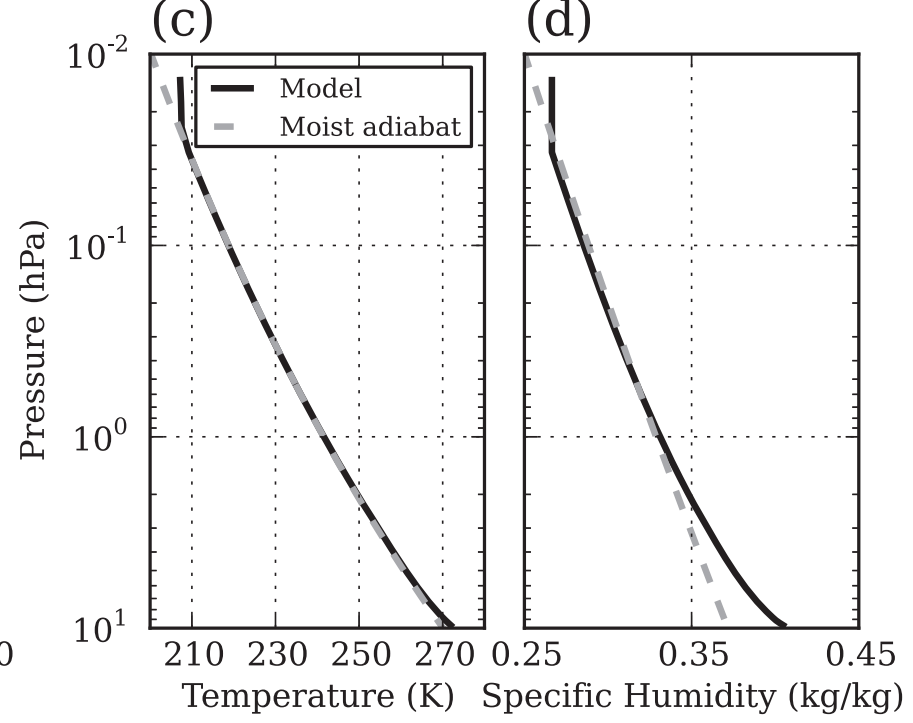

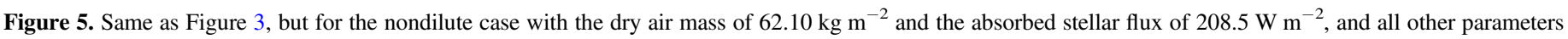
kept the same as in the dilute simulation.

convective warming is located at $\sim 500 \mathrm{hPa}$, slightly higher than where most of the condensate forms.

In this $1 \mathrm{D}$ column model, the water vapor profile is only updated in the surface evaporation and convection scheme. Therefore, in the steady state, the water vapor concentration does not change during the convective adjustment, except at the lowest model layer. In this layer, convective transport of moisture upward dries the layer (Figure 4(b)), which is balanced by moisture input from evaporation. An equivalent amount of water vapor then condenses out as air parcels rise in the atmosphere (Figure 4(c)), and falls to the ground, closing the mass budget of the climate system.

\subsection{Nondilute Simulation}

With an Earth-like noncondensible inventory and $\tau_{0}=1.2$, the atmosphere does not become strongly nondilute until the stellar flux is sufficiently high to cause a runaway, in which case the system does not reach equilibrium. In order to illustrate strongly nondilute convection in equilibrium, one could increase $\tau_{0}$ with fixed stellar flux, or keep $\tau_{0}$ at its original value while reducing the noncondensible inventory. Here we choose the latter approach and raise the water vapor concentration in the 1D model by reducing the mass of the dry air to $62.10 \mathrm{~kg} \mathrm{~m}^{-2}$. In this case, the atmosphere can be nondilute even at the freezing point of water since the saturation vapor pressure of water in the atmosphere only depends on the air temperature. This provides a simple and clean test of energy and mass conservation in the nondilute case. With reduced noncondensible pressure, the curve $\operatorname{OLR}\left(T_{s}\right)$ shows very little overshoot; OLR $\infty$ is the same as in the previous case, as it is determined by the limiting case of a pure water vapor atmosphere. The absorbed stellar flux in this case was set at $208.5 \mathrm{~W} \mathrm{~m}^{-2}$, which is just short of the value at which the system enters a runaway state.

In this nondilute case, the system reaches both energy and mass equilibrium after integration of 3000 days (Figures 5(a) and (b)). The net evaporation rate at the surface approaches zero exponentially with time, similar to the dilute case (Figure 3(b)). However, the net radiative flux at the top of the model evolves somewhat more slowly compared with the dilute simulation (Figure 3(a)). This is related to the high climate sensitivity of water-rich atmospheres, i.e., the low slope of OLR as a function of surface temperature. Figures 5(c) and (d) show the equilibrium temperature and humidity profiles. The convection is so deep that it establishes a moist adiabat nearly throughout the column atmosphere. The tropopause is close to the top of the column model, $\sim 0.03 \mathrm{hPa}$. The humidity profile confirms that water vapor is nondilute in the atmosphere. Corresponding to the thermal structure, the vertical distribution of the specific humidity becomes more uniform compared with the dilute case. Even at the top of the model, the specific humidity is as high as $0.27 \mathrm{~kg} \mathrm{~kg}^{-1}$. In addition, the surface pressure in equilibrium is about $9.75 \mathrm{hPa}$ (Figures 5(c) and (d)), so that the mass of water in the atmosphere is $\sim 40 \mathrm{~kg}$, approximately two-thirds of that of the dry air. The humidity near the ground is slightly in excess of the value associated with the saturated moist adiabat, but this does not actually arise from supersaturation in the convection scheme. Rather, the excess moisture arises from the fact that the temperature profile is very slightly warmer than the moist adiabat and that the saturated specific humidity is very sensitive to temperature when the noncondensible inventory is so low.

The vertical structure of condensation and convective heating for nondilute convection will be discussed in connection with the runaway greenhouse simulation.

\subsection{Runaway Greenhouse and Seasonal Cycle Simulation}

To test how the moist convection scheme behaves for timedependent simulations, we use the 1D column model to simulate the runaway greenhouse atmosphere on a planet with a high eccentricity $(e=0.5)$. The orbital period of the planet in this simulation is 50 days, and the stellar flux at the distance equal to the semimajor axis of the orbit is $238 \mathrm{~W} \mathrm{~m}^{-2}$. Hence, the average of the stellar flux received by the planet over the course of the planetary year is $238 / \sqrt{1-0.5^{2}}=274.82 \mathrm{~W} \mathrm{~m}^{-2}$, which is sufficiently high to force the system into a runaway. Kepler's second law indicates that the planet travels slowly near the apastron 


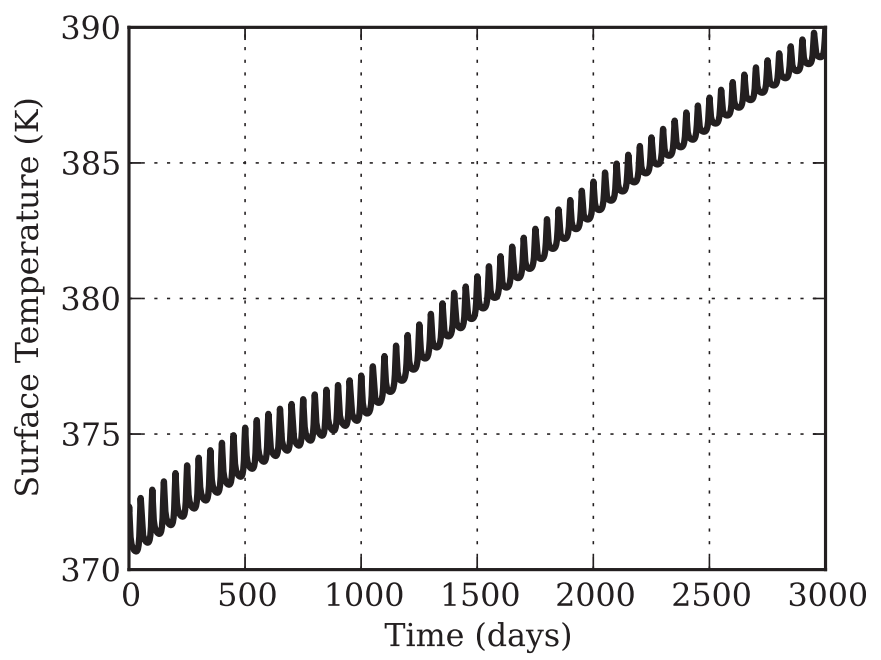

Figure 6. Time evolution of the surface temperature in the runaway greenhouse simulation. The seasonal cycle of the surface temperature is very weak $(\sim 1.5 \mathrm{~K})$ despite the highly eccentric orbit assumed.

(winter) and rapidly during periastron (summer), which would lead to short, hot summers and long, cold winters on a planet with little thermal inertia.

We integrated the model for 3000 days starting from an initial surface temperature of $372 \mathrm{~K}$. The surface temperature evolution over this time period is shown in Figure 6. The temperature exhibits a steady upward trend rising by $\sim 20 \mathrm{~K}$ over the course of the integration, with only a small superposed seasonal cycle. Because the annual mean OLR (Figure 7(a)) remains near the limiting value of $210 \mathrm{~W} \mathrm{~m}^{-2}$, which is less than the absorbed solar radiation, the planet is in a runaway state and the temperature will continue to grow indefinitely so long as a liquid ocean remains to feed the increasing atmospheric water content. Because the surface temperature response shows only a weak seasonal cycle, the runaway conditions are little affected by the strong seasonal cycle of insolation, even though the planet undergoes a long winter with stellar flux as low as $100 \mathrm{~W} \mathrm{~m}^{-2}$ (gray solid line in Figure 7(a)). If it were not for the evidently strong thermal inertia of the system, much of the water vapor evaporated in summer would condense back onto the surface in the long winter.

The detailed response of the surface temperature to the large seasonal stellar forcing is shown in Figure 7(a). The surface temperature varies by only $1.5 \mathrm{~K}$, in spite of large stellar flux variation from 900 to $100 \mathrm{~W} \mathrm{~m}^{-2}$ and small surface thermal inertia (recall that the thickness of the slab ocean is only $1 \mathrm{~m}$ ). The surface energy budget in the $1 \mathrm{D}$ model is

$$
\frac{d\left(\rho_{w} c_{p w} H T_{s}\right)}{d t}=-\mathrm{SW}-\mathrm{LW}-\mathrm{SH}-\mathrm{LH}+\left(E_{p}-E_{e}\right) .
$$

Here $\rho_{w}$ and $c_{p w}$ are the density and specific heat of liquid water, respectively, $H=1 \mathrm{~m}$ is the thickness of the slab ocean, SW and LW are the net shortwave and longwave radiative fluxes (positive when going upward), $\mathrm{SH}$ and $\mathrm{LH}$ are sensible and latent heat fluxes at the surface, respectively, $E_{p}$ is the energy loss of the atmosphere when the condensate falls to the ground (equivalent to the sum of the internal energy and potential energy of the precipitated water, or the static energy of the precipitated water), and $E_{e}$ is the energy increase of the atmosphere when water goes into the atmosphere by evaporation (equivalent to the sum of the internal energy and potential energy of the evaporated water, or the static energy of the evaporated water). In the runaway greenhouse simulation, both LW and $\mathrm{SH}$ are small because the surface air temperature stays very close to the surface temperature and the atmosphere is optically thick for longwave radiation near the ground. Hence, the absorbed stellar radiation is nearly balanced by the energy flux associated with the phase transition of water $\left(\mathrm{LH}+E_{e}-E_{p}\right.$; see Figure 7(b)). Therefore, most of the stellar flux is used to change the mass of the atmosphere instead of the surface temperature. The phase transition of water strongly damps temperature fluctuations, as it takes only a small change in surface temperature to drive a large change in the energy fluxes associated with water when the water content of the atmosphere is so high. Hence, the planet exhibits a very weak seasonal cycle in spite of its high-eccentricity orbit. Note that $E_{e}-E_{p}$ is of comparable magnitude to the latent heat flux, so that this term makes up a crucial part of the exchange of energy between surface and atmosphere in the strongly nondilute case.

Although the thermal inertia associated with phase change strongly damps the seasonal cycle, it is easy to verify from a simple energy balance argument that this thermal inertia does not cause a significant delay in transfer of water from the ocean to the atmosphere. For very hot conditions, the atmospheric energy storage is dominated by water vapor. As an upper bound, let's estimate the energy of a saturated atmosphere with surface temperature and pressure at the critical point of water, beyond which point the distinction between ocean and atmosphere disappears. The mass of the atmosphere per unit area of planetary surface is $p_{\text {crit }} / g$, where $p_{\text {crit }}$ is the critical point pressure. As an upper bound to latent heat storage, we multiply by the latent heat $L$ at 1 bar and $373 \mathrm{~K}$, which overestimates the latent heat storage because in reality $L$ approaches zero as the critical point is approached (an effect not currently incorporated in our convection scheme). Besides the latent heat storage, the atmosphere stores dry enthalpy at a rate $c_{p} T$ per unit mass, where $T$ is the mass-weighted temperature of the atmosphere. As an upper bound to this, we take $T=T_{\text {crit }}$, which is not a bad approximation given the slow logarithmic decay of $T(p)$ for a pure steam atmosphere. The estimated energy storage per unit area is then $E=\left(L+c_{p} T_{\text {crit }}\right) p_{\text {crit }} / g$, which works out to $8 \times 10^{12} \mathrm{~J} \mathrm{~m}^{-2}$ for Earth-like gravity, with specific heat taken at the critical point. The net flux available to allow this energy to accumulate is the difference between the incoming stellar radiation and the $\mathrm{OLR}_{\infty}$, which is $64 \mathrm{~W} \mathrm{~m}^{-2}$. Dividing this into $E$, the time needed to reach the critical point is under 4000 Earth years, which is short compared to the other processes involved in irreversible water loss. The time required to reach the critical point becomes infinite as the absorbed stellar flux approaches $\mathrm{OLR}_{\text {crit }}$, but even reducing the excess flux by a factor of 100 would not bring the thermal inertia delay into the range where it can be considered a significant inhibition to the runaway greenhouse process.

The vertical structure of the temperature and specific humidity on the last day of simulation is shown in Figures 7(c) and (d). For the runaway greenhouse simulation, water vapor is nondilute in the atmosphere. Similar to the 
(a)

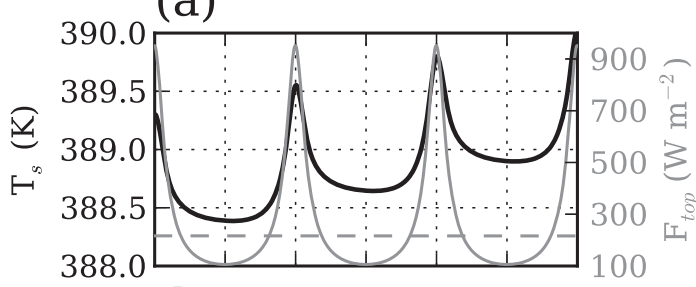

(b)

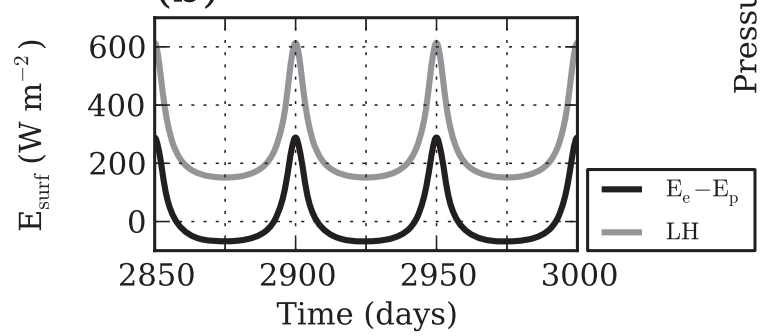

(c)

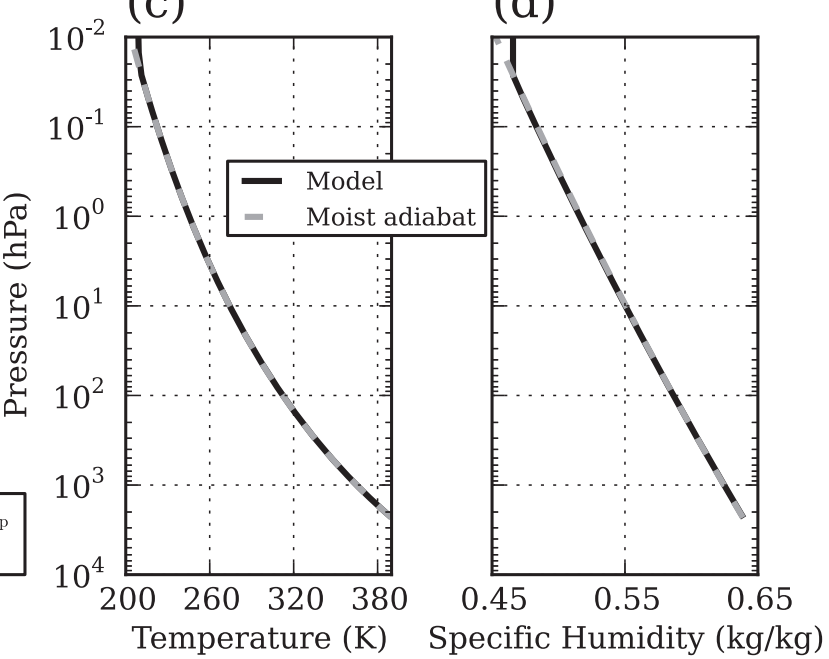

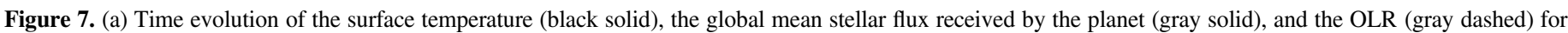

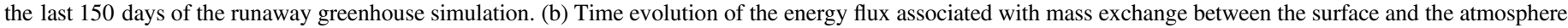

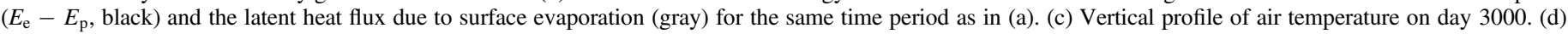
Vertical profile of the specific humidity on day 3000.

nondilute simulation in Section 3.3, essentially the whole atmosphere is subject to convective adjustment. As the surface temperature reaches $390 \mathrm{~K}$, water vapor is nondilute even in the upper atmosphere above $10 \mathrm{hPa}$, leading to large infrared opacity there. In contrast to the dilute simulation discussed in Section 3.2 (Figure 4), condensation and convective heating are concentrated in a thin layer in the upper atmosphere, from 0.1 to $10 \mathrm{hPa}$. This is in part due simply to the optical thickness of the atmosphere, which implies that the strong radiative cooling needed to balance the latent heat release due to strong condensation can only occur in the high portions of the atmosphere, where the atmosphere first begins to become optically thin and from which infrared can escape to space. However, a full understanding of the situation in the nondilute case requires some discussion of vertical motion and buoyancy generation, which exhibit key differences from the dilute case, which will be discussed in the next section.

\section{DISCUSSION}

\subsection{Vertical Motion Induced by Precipitation}

The fact that precipitation carries a significant mass flux has important implications for the vertical motion of the gas-phase part of the atmosphere in the nondilute case. Vertical motion is diagnosed from the continuity equation. In the nondilute case, the continuity equation needs to be modified, taking the mass loss due to precipitation into account. Specifically, assuming the hydrostatic approximation,

$$
\partial_{p} \omega+\nabla \cdot(v)=P,
$$

where $v$ is the horizontal velocity and $P$ is the rate of mass precipitation, per unit mass of the atmosphere. This is also the form the continuity equation would take when incorporating the convection scheme into a nondilute 3D GCM. This effect is included in Leconte et al. (2013) in their study of the runaway greenhouse threshold of the Earth's climate system. Integrating the continuity equation from the top of the atmosphere shows that a vertical motion is induced when the condensate is removed from the atmosphere ( $\omega_{2}$ in Equation (24)):

$$
\begin{aligned}
\omega(p) & =\omega_{1}+\omega_{2} \\
\omega_{1} & \equiv-\int_{0}^{p} \nabla \cdot v_{p} d p \\
\omega_{2} & \equiv \int_{0}^{p} P d p .
\end{aligned}
$$

Here $\omega_{1}$ comes from the air flow divergence above the pressure level, and $v_{p}=(u, v)$ is the horizontal wind velocity along the isobaric surface and vanishes in the 1D model simulation since there is no horizontal flow; this term would be present in a 3D model. In the dilute limit there would be no mean vertical motion in an isolated 1D column, but in the nondilute case a mean gaseous vertical motion $\omega_{2}$ is supported by the downward mass flux of precipitation. Figure 8(c) shows the vertical velocity $\omega_{2}$ due to the removal of condensed water from the atmosphere for the runaway greenhouse simulation and indicates that some condensation also occurs between the 1 bar level and the surface. In this layer, the latent heat release is balanced by the adiabatic cooling due to the upward motion. For our single-column model, the way that the vertical mass flux is balanced by a return mass flux in the form of precipitation can be considered as a peculiar form of a onecolumn Hadley cell, in which the return mass flow compensating the upward motion in the convecting column is balanced by downward mass flux due to precipitation. For a conventional Hadley cell, the upward mass flux consists primarily of noncondensible gas and must be balanced instead by downward noncondensible mass flux in adjoining subsiding regions, which leads to compressional heating there. The magnitude of vertical motion in the optically thick nondilute case is small, however, compared with the typical large-scale vertical velocity in the Intertropical Convergence Zone (ITCZ) of present Earth $\left(\sim 0.1 \mathrm{~Pa} \mathrm{~s}^{-1}\right)$. It is important to note that the transport of moisture and heat due to $\omega_{2}$ does not need to be incorporated in the column model or 3D GCM as an explicit 
(a)

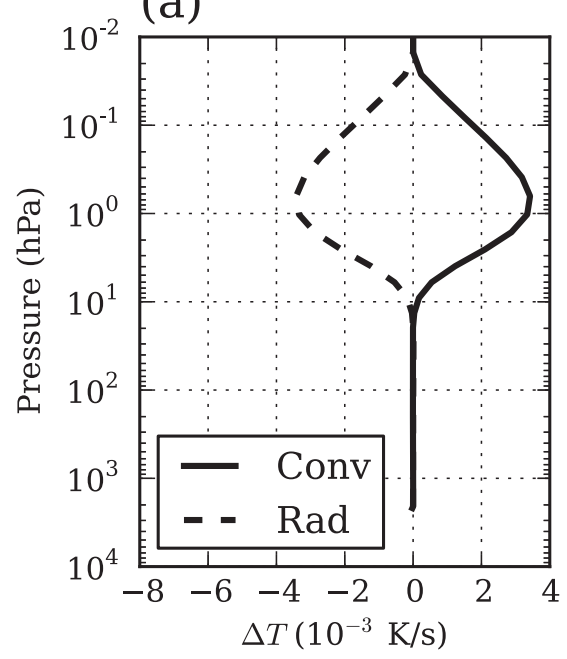

(b)

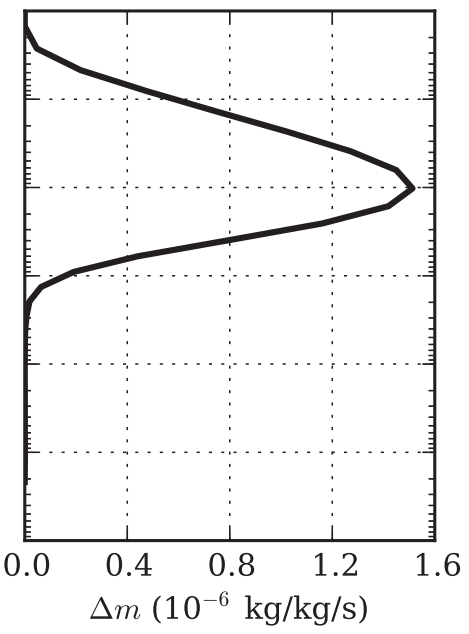

(c)

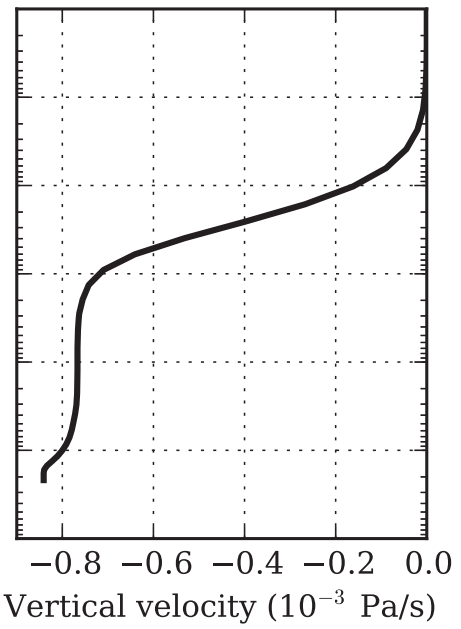

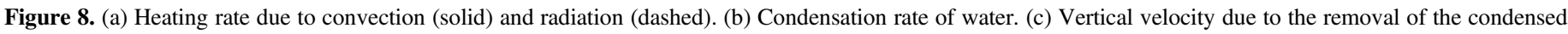
water from the atmosphere. All data are from the runaway greenhouse simulation on day 3000 .

vertical advection term. This transport is handled implicitly as part of the convective adjustment process. Specifically, it is manifest as the change in pressure that occurs in a layer when mass is removed from a higher layer by precipitation.

\subsection{Buoyancy Generation in Dilute versus Nondilute Atmospheres}

In the dilute limit, absorption of stellar radiation at the surface heats the low-lying air, which then picks up moisture from the adjacent surface; this builds buoyancy near the surface, leading to deep convection that takes the form of condensing plumes penetrating deep into the atmosphere. In the highly nondilute limit, in contrast, it is not possible to create buoyancy in this way, because the saturated moist adiabat collapses onto a unique curve without free parameters-the dewpoint/frostpoint formula obtained by solving the ClausiusClapeyron relation for $T(p)$-which is neutrally stable with regard to pseudoadiabatic vertical displacements. In contrast, in the dilute limit, the lower atmosphere can be heated to a different member of the moist adiabatic family of profiles, which is buoyant with regard to the overlying atmosphere. In the strongly nondilute case, heating the lower atmosphere increases surface pressure instead of buoyancy, in effect adding nonbuoyant mass at the bottom of the atmosphere. Similarly, it is difficult to generate top-driven convection through production of negative buoyancy by radiative cooling in the upper atmosphere, because the energy loss goes into atmospheric mass loss via precipitation, which reduces surface pressure rather than generating negative buoyancy aloft. (Retention of condensate would alter this conclusion.) The lack of buoyancy generation in single-component condensible atmospheres was noted in Colaprete \& Toon (2003) in connection with saturated pure $\mathrm{CO}_{2}$ Martian convection, but it is in fact a generic property of nondilute convection.

Our single-column simulations shed some further light on how nondilute moist convection works in the absence of buoyancy generation. Table 1 gives the ratio of the water vapor transported upward from the lowest layer during moist convection to the total evaporated water added to the layer during surface evaporation, which measures the buoyancy of the atmosphere during moist convection. This ratio is smaller than unity. The remaining part stays in the lowest layer, increasing the mass of the atmosphere, with negligible amounts condensing out there (see Figures 4(c) and 8(b)). In our simulations, this ratio is nearly the same as the mass concentration of noncondensible substance in the near surface layer $\left(1-q_{s a}\right)$, as shown in the table. This simple relationship is confirmed by numerical simulations with a variety of different surface air specific humidities $\left(0<q_{s a}<0.95\right.$, not shown). For the Earth-like atmosphere where water vapor is dilute, nearly all of evaporated water vapor is transported upward and forms precipitation in the mid-troposphere, releasing latent heat that balances the IR cooling there (Figure 9(a)). The column model indicates that the buoyancy of the atmosphere is getting weaker as water vapor becomes dominant in the atmosphere, with less vertical transport by deep convective plumes that nearly instantly mix water vapor throughout the depth of the troposphere. The evaporated water vapor tends to mostly stay in the lowest layer.

In the runaway simulation, where the mass of the atmosphere is steadily increasing, most of the evaporated water does indeed simply stay where it is put, at the bottom of the atmosphere. In an equilibrium situation such as the cold nondilute simulation with reduced noncondensible pressure, the surface pressure eventually stops growing, and so the mass added to the lowest model layer by evaporation must be carried away by some other means than deep convection. The answer lies in the advection due to the vertical velocity $\omega_{2}$, which carries moisture just a little ways upward from the lowest layer, rather than distributing it through the depth of the troposphere as deep convection would do. The equilibrium mass budget in strongly nondilute convection consists of addition of moisture to the bottom of the atmosphere by evaporation, which is then advected upward to the layer where the mass can condense out and return to the surface as precipitation. From the standpoint of energetics, the reduction in surface pressure due to precipitation must balance the increase due to evaporation because condensation is determined by infrared radiative cooling to space, while evaporation is determined by absorption of stellar radiation at the surface, and the two energy fluxes must balance in equilibrium. The contrast between dilute and 
Table 1

List of Some Surface and Atmospheric Variables in the Three Numerical Simulations Discussed in Section 3

\begin{tabular}{|c|c|c|c|c|}
\hline Surface and Atmospheric Variables & Dilute & Nondilute & Runaway (Summer) & Runaway (Mean) \\
\hline Surface temperature $(\mathrm{K})$ & 306.29 & 279.89 & 390.04 & 388.85 \\
\hline$E_{p}-E_{e}\left(\mathrm{~W} \mathrm{~m}^{-2}\right)$ & -2.75 & 2.91 & -288.87 & -3.89 \\
\hline$\Delta F_{\text {top }}-\Delta F_{\text {bottom }}\left(\mathrm{W} \mathrm{m}^{-2}\right)$ & 186.23 & 150.25 & 190.02 & 200.94 \\
\hline$f($ water $)\left(\mathrm{kg} \mathrm{kg}^{-1}\right)$ & 0.980 & 0.596 & 0.352 & 0.359 \\
\hline
\end{tabular}

Note. For the runaway case, values are given both for the summer at day 3000 of the simulation and for the average over the last seasonal cycle. $\left(E_{p}-E_{e}\right)$ is the energy flux associated with the mass exchange between the atmosphere and surface, $\left(\Delta F_{\text {top }}-\Delta F_{\text {bottom }}\right)$ is the longwave radiative flux leaving the air column, $q_{s a}$ is the specific humidity of the surface air, and the ratio $f$ (water) measures the portion of water vapor transported upward from the lowest layer by the moist convection relative to the total evaporated water added to the layer by the surface evaporation.

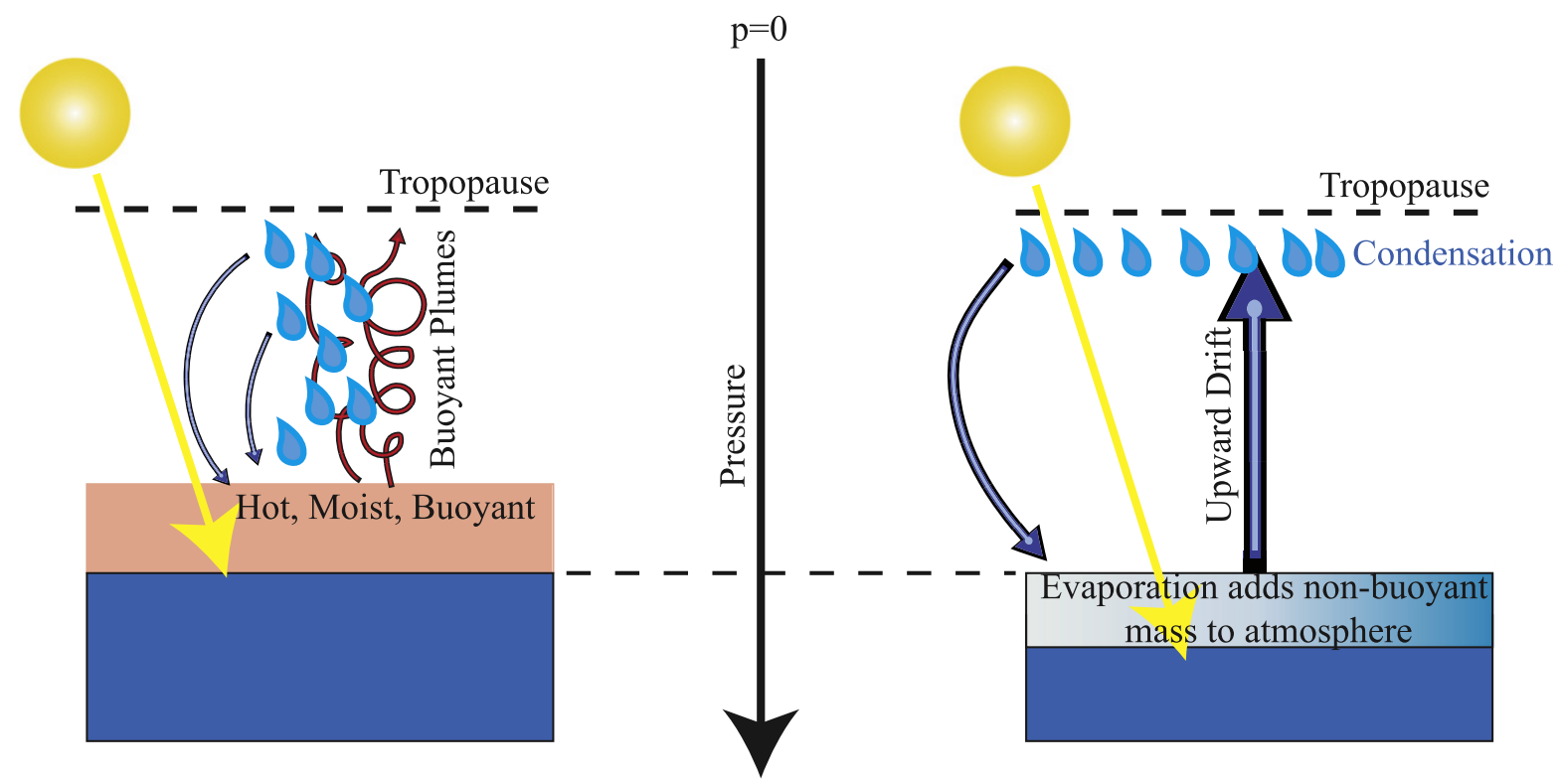

Figure 9. Schematic of moist convection in Earth-like conditions with a dilute atmosphere that is not optically thick throughout the infrared (left) and for a strongly nondilute optically thick atmosphere (right). In the Earth-like case, shortwave stellar energy absorbed at the ocean surface leads to the creation of a hot moist layer of air near the surface, which is buoyant with respect to the overlying atmosphere and results in deep convection. In the strongly nondilute case, stellar heating instead adds mass to the bottom of the atmosphere in the form of condensible vapor. Because of constraints related to Clausius-Clapeyron, the new mass added is not buoyant with regard to the overlying atmosphere and is only gradually carried upward by gradual laminar ascent, to upper layers of the troposphere where the latent heat released by rapid condensation can be balanced by infrared cooling to space.

nondilute moist convection is summarized in Figure 9. In the nondilute case convection takes the form of a "moisture elevator" in which moisture added at the surface ascends the floors of the atmosphere in an orderly and gradual process, in contrast to the chaotic, turbulent process by which deep convection transports moisture in the dilute case. In the nondilute case, a mean vertical motion can exist in a single column, with the upward vapor-phase mass flux balanced by downward mass flux from precipitation and the condensational heating balanced locally by radiative and adiabatic cooling; this is another manifestation of the singlecolumn Hadley circulation described previously. For the dilute case, in contrast, any mean upward motion in a column can still balance condensational heating against adiabatic cooling by ascent locally, but the upward noncondensible mass flux must be compensated by subsidence in the surrounding air, which leads to compressional heating that must be balanced by radiative cooling there.

\subsection{Energy Transport by Precipitation, and Precipitation- Temperature Scaling}

The significant energy carried by precipitation is one of the novel features of nondilute convection. For present Earth's climate, even though the precipitation rate is trivial compared with the mass of the atmosphere, $E_{p}$ is a large value. For a typical value of precipitation rate in the tropics $\left(5 \times 10^{-5} \mathrm{~kg} \mathrm{~m}^{-2} \mathrm{~s}^{-1}\right), E_{p}$ is as high as $50 \mathrm{~W} \mathrm{~m}^{-2}$. Table 1 shows $E_{p}-E_{e}$ for the three simulations in Section 3. The column for the dilute simulation, which is carried out in Earthlike conditions, shows that even though most of $E_{p}$ is canceled by the internal energy of the liquid water evaporated from the surface, the remaining part $\left(E_{p}-E_{e}\right)$ is still not entirely negligible, attaining a value of approximately $-2.75 \mathrm{~W} \mathrm{~m}^{-2}$. For the cold nondilute simulation with reduced noncondensible mass, the value gets slightly larger in magnitude and changes sign. The change in sign arises primarily because the 


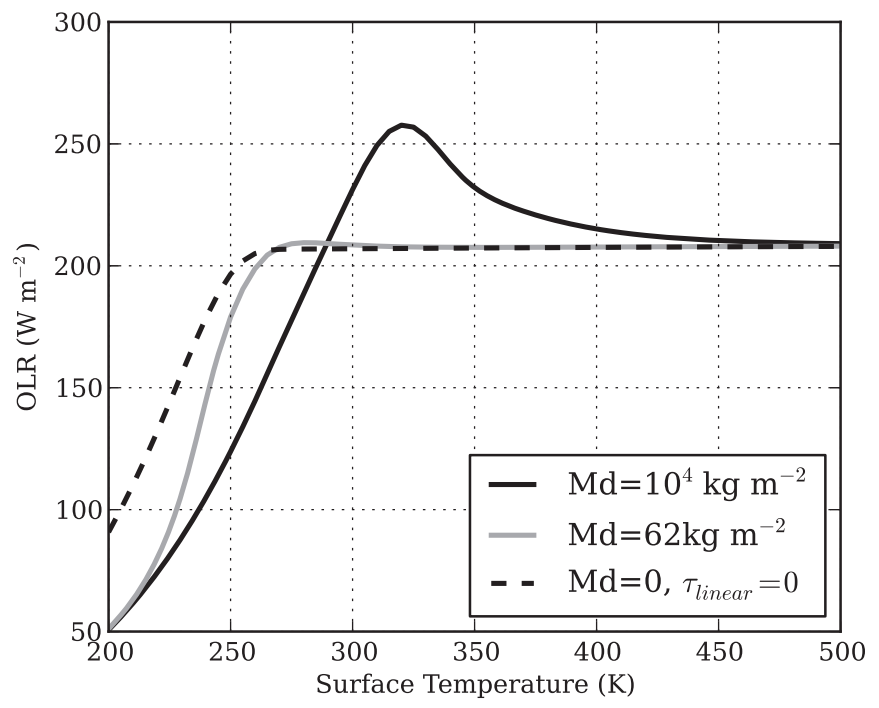

Figure 10. OLR vs. surface temperature for a saturated moist adiabatic atmosphere using the same gray-gas radiation model as used in the calculations described in the text. $M_{d}$ is the mass path of the noncondensible dry background gas. The two cases with nonzero $M_{d}$ are calculated with background optical thickness $\tau_{0}=1.2$. The dashed line gives results for a pure water vapor atmosphere with no background temperature-independent opacity.

precipitation is warmer, owing to the weaker vertical gradient of temperature in the nondilute case. Even in the hot runaway case, the seasonal mean value only increases modestly in magnitude, to $-3.89 \mathrm{~W} \mathrm{~m}^{-2}$. The modest values of $E_{p}-E_{e}$ trace back to the fact that the precipitation rate is similar in all three cases, with only moderate increases even in the hot runaway case. In equilibrium or near-equilibrium, this limits the mass available to carry energy. In the course of the seasonal cycle, however, the exchange term can be very large, as evidenced by the summer runaway value in Table 1 . In that case, $E_{p}-E_{e}$ is comparable to the absorbed solar flux at the surface. In our idealized calculation, all the energy carried by the precipitation is deposited at the ground, but in reality some proportion would be transferred to the atmosphere by evaporation and frictional dissipation on the way down.

The limited precipitation rate noted above arises from energetic limitations already familiar from studies of Earth's climate. Because of limitations on turbulent transfer of sensible heat through a stable boundary layer, the net evaporation (and hence, in equilibrium, net precipitation) cannot much exceed the stellar radiation reaching the surface (Pierrehumbert 2002; Le Hir et al. 2009). For the runaway case the limit is $1.1 \times 10^{-4} \mathrm{~kg} \mathrm{~m}^{-2} \mathrm{~s}^{-1}$, only slightly in excess of the realized precipitation. Another way of looking at the energetics is that the dominant balance in the troposphere when sufficiently large amounts of condensible substance are present is between latent heat release and net radiative cooling (which is purely infrared in our case). The column-integrated radiative cooling is shown in Table 1. It accounts well for the precipitation in the two optically thick nondilute cases, translating into a precipitation rate of $6.07 \times 10^{-5} \mathrm{~kg} \mathrm{~m}^{-2} \mathrm{~s}^{-1}$ in the cold nondilute case and $9.04 \times 10^{-5} \mathrm{~kg} \mathrm{~m}^{-2} \mathrm{~s}^{-1}$ in the runaway case. The radiative cooling limit significantly overestimates the precipitation in the dilute case, however, because in that case much of the radiative cooling is balanced instead by sensible heat transport due to convection.

\section{CONCLUSIONS}

We have developed a simple energy-conserving convection parameterization that works across atmospheres ranging from those in which the condensible substance is a dilute component of the atmosphere to those with strongly nondilute condensibles. The parameterization is currently limited to a single condensible substance in a noncondensible background mixture of gases, but it can be applied to any combination of the two classes of components, given suitable adjustment to thermodynamic parameters. The performance of the parameterization has been tested within a single-column radiativeconvective model, but it is designed to be suitable for incorporation in GCMs. Simulations of that nature are under way and will be reported on in a future paper. Although our parameterization is not the first attempt to treat atmospheres with nondilute condensibles, we have endeavored to use the formulation of the parameterization as a vehicle to give a thorough discussion of the nature of energy conservation in nondilute atmospheres and the various ways in which moist convection in such atmospheres differs from the more familiar dilute limit. Key features include transport of significant amounts of energy and mass by precipitation (especially in the course of the seasonal cycle when the atmosphere is out of equilibrium), the suppression of deep buoyant plumes in favor of a more gentle gradual ascent, and the feedback of precipitation and evaporation/sublimation on surface pressure (a feature already familiar from the current Martian atmosphere). The single-column simulations were conceived primarily as a test of the conservation properties of the scheme, but in addition to illustrating some general features of nondilute convection, they demonstrated that nondilute atmospheres can have a very strong damping effect on seasonal cycles driven by even extreme seasonal variations in instellation.

In principle, the large proportion of condensible substance present in nondilute atmospheres could lead the energy transport by condensate to dominate the behavior of the system. In all the cases we have examined, however, the energy carried by condensate, while a significant term needed to enforce energy conservation, is of fairly modest magnitude in the annual mean. This property arises from energetic limits on precipitation rate: in essence, one cannot release latent heat at a rate faster than it can be resupplied by absorption of stellar radiation, and so the large latent heats of most condensible substances tend to yield limited mass of precipitation. This is a global constraint, which exerts dominant control in a singlecolumn model, but it would not apply locally in 3D simulations, so the incorporation of energy transport by condensate may have more dramatic effects when a scheme such as ours is embedded in a 3D GCM.

The convection scheme we have developed adjusts the atmosphere to a saturated pseudoadiabat, using the lapse rate in comparison to the nondilute pseudoadiabat as the sole criterion for convection. This achieves an equivalent of what is commonly done in single-column radiative-convective models and thus leads the way for GCM experiments focusing on dynamical effects in nondilute atmospheres. When dealing with a situation presenting the novel physics of nondilute convection, it is useful to revert to simplified schemes such as this, which are easy to understand, and which can most easily be made to incorporate the most fundamental physical constraints. This approach complements approaches such as that pursued by Wolf \& Toon (2015), in which an attempt is made to adapt 
the complex Zhang-Macfarlane convection scheme-which incorporates many empirical assumptions based on Earth's current atmosphere - to nondilute conditions. However, unconditional convective adjustment to a pseudoadiabat does not necessarily represent the way convection operates in reality. There are many potentially important physical effects that have not been taken into account. Among other things, the derivation of the convection scheme has revealed a currently unconstrained parameter governing the distribution of condensate following convection, and there is currently no good physical basis for setting this parameter. In addition, more work is needed on the actual behavior of the shallow nonprecipitating convection that occurs when there is insufficient condensible to allow the adjusted state to be saturated, as well as on schemes that in general allow the adjusted state to be subsaturated. Further, given possibly strong contrasts between the molecular weight of the condensible and that of the background gas, there is a need to incorporate compositional effects on buoyancy into the parameterization, along the lines explored for $\mathrm{H}_{2}-\mathrm{H}_{2} \mathrm{O}$ atmospheres by Li \& Ingersoll (2015). Such effects would be particularly extreme for $\mathrm{H}_{2}$ atmospheres incorporating condensible $\mathrm{CO}_{2}$ from a surface $\mathrm{CO}_{2}$ ocean or glacier and would yield stable layers near the ground that are highly resistant to the initiation of convection. Some insights may be gained by studying convection in nondiute atmospheres in the solar system - notably those of Mars and Titan-but it is likely that further development of nondilute convection parameterizations will need to be informed by simulations with resolved 3D convection/cloud simulations.

We thank Robin Wordsworth, Dorian Abbot, Daniel Koll, and Jun Yang for many helpful discussions. Support for this work was provided by the NASA Astrobiology Institutes Virtual Planetary Laboratory Lead Team, under the National Aeronautics and Space Administration solicitation NNH12ZDA002C and Cooperative Agreement Number NNA13AA93A.

\section{APPENDIX \\ ENERGY CONSERVATION FOR NONDILUTE SYSTEMS}

The following closely follows the derivation given in Trenberth (1997), but with close attention paid to retention of terms that are typically dropped in the dilute limit.

We begin with the momentum and mass continuity equations, and the first law written in the form given in Equation (8). In altitude ( $z$ ) coordinates these take the form

$$
\begin{gathered}
\rho \frac{d}{d t} \boldsymbol{v}=-\nabla p-\rho g \hat{z} \\
\partial_{t} \rho+\nabla \cdot \rho \boldsymbol{v}=0 \\
\frac{d}{d t}\left(k-\frac{p}{\rho}\right)+p \frac{d}{d t} \frac{1}{\rho}=Q
\end{gathered}
$$

where $d / d t$ is the material derivative. The density $\rho$ includes both the gas-phase and condensed-phase mass; mass may exchange between gas and condensed phase, but it is presumed in this derivation that no mass leaves the air parcel in which it was originally found and that the temperature of the condensate is the same as the temperature of the surrounding gas. The effect of relaxing these assumptions is discussed at the end of this appendix. $Q$ is the diabatic heating rate per unit mass.
Taking the dot product of the momentum equation (Equation (25)) with $\boldsymbol{v}$ and using the mass continuity equation (Equation (26)) yields

$$
\partial_{t} \frac{1}{2} \rho v \cdot v+\nabla \cdot\left(\frac{1}{2} \rho v \cdot v\right) v=-v \cdot \nabla p-\rho g w
$$

where $w$ is the vertical velocity. Using $w=d z / d t$ together with the continuity equation allows us to write the following expression for the evolution of kinetic plus potential energy:

$$
\partial_{t}\left(\frac{1}{2} \rho v \cdot v+\rho g z\right)+\nabla \cdot\left(\frac{1}{2} \rho v \cdot v+\rho g z\right) v=-v \cdot \nabla p .
$$

Using the mass continuity equation, the right-hand side can be rewritten as

$$
-\boldsymbol{v} \cdot \nabla p=-\nabla \cdot p v+\frac{p}{\rho} \rho \nabla \cdot v=-\nabla \cdot p v-\frac{p}{\rho} \frac{d \rho}{d t} .
$$

Now, upon multiplying by $\rho$ the first law in Equation (27) can be rewritten as

$$
\partial_{t} \rho\left(k-\frac{p}{\rho}\right)+\nabla \cdot \rho\left(k-\frac{p}{\rho}\right) \boldsymbol{v}-\frac{p}{\rho} \frac{d}{d t} \rho=\rho Q
$$

which can be combined with Equations (29) and (30) to yield the conservation law

$$
\partial_{t} \rho \mathcal{E}+\nabla \cdot \rho \mathcal{F}=\rho Q
$$

where the energy density is

$$
\mathcal{E} \equiv \frac{1}{2} \boldsymbol{v} \cdot \boldsymbol{v}+g z+\left(k-\frac{p}{\rho}\right)
$$

and the energy flux is

$$
\mathcal{F} \equiv\left(\frac{1}{2} \boldsymbol{v} \cdot \boldsymbol{v}+g z+k\right) \boldsymbol{v} .
$$

If the kinetic energy is negligible, the conservation law in the form of Equation (9) follows upon integrating over an isolated column of the atmosphere.

The above calculation suffices to demonstrate moist enthalpy conservation in the context of the convective adjustment protocol employed in the text, in which a layer is first mixed with condensate retained, followed by a separate step in which condensate is removed from the column, taking its enthalpy and potential energy with it. In a real atmosphere, condensate would be redistributed among air parcels continuously, with some particles eventually reaching the bottom, where they are removed. A formal treatment of conservation in this situation would be very complex, given that each condensate particle has its own history-dependent velocity and each air parcel retains a mix of condensate particles each with their own characteristics. However, a simple physical argument suffices to justify conservation in the general case. Imagine a situation in which a single condensate droplet moves from one air parcel to an adjoining one. In that case, it simply carries its own enthalpy with it, so the enthalpy removed from one air parcel is added to the other, and similarly, potential and kinetic energy is moved, with some of the mechanical energy left behind in the source air parcel if there has been drag against the particle, and some interchange between potential and kinetic energy if the particle 
has changed altitude. Subsequent transformations of the condensate without leaving the destination parcel are within the scope of the preceding derivation. Since the energy transport for each droplet occurs independently of the others, if conservation applies for displacement of an individual droplet, it will also apply for an ensemble of droplets.

\section{REFERENCES}

Betts, A. K. 1986, QJRMS, 112, 677

Betts, A. K., \& Miller, M. J. 1986, QJRMS, 112, 693

Colaprete, A., \& Toon, O. B. 2003, JGRE, 108, 5025

Emanuel, K. A. 1994, Atmospheric Convection (Oxford: Oxford Univ. Press)

Forget, F., Hourdin, F., \& Talagrand, O. 1998, Icar, 131, 302

Frierson, D. M. W. 2007, JAtS, 64, 1959

Ingersoll, A. P. 1969, JAtS, 26, 1191

Ishiwatari, M., Takehiro, S.-i., Nakajima, K., \& Hayashi, Y.-Y. 2002, JAtS, 59,3223

Kasting, J. F. 1988, Icar, 74, 472

Kasting, J. F. 1991, Icar, 94, 1

Kasting, J. F., Whitmire, D. P., \& Reynolds, R. T. 1993, Icar, 101, 108
Kopparapu, R. K., Ramirez, R., Kasting, J. F., et al. 2013, ApJ, 765, 131

Le Hir, G., Donnadieu, Y., Goddéris, Y., et al. 2009, E\&PSL, 277, 453

Leconte, J., Forget, F., Charnay, B., Wordsworth, R., \& Pottier, A. 2013, Natur, 504, 268

Li, C., \& Ingersoll, A. P. 2015, NatGe, 8, 398

Manabe, S., Smagorinsky, J., \& Strickler, R. F. 1965, MWRv, 93, 769

Manabe, S., \& Strickler, R. F. 1964, JAtS, 21, 361

Merlis, T. M., \& Schneider, T. 2010, JAMES, 2, 13

Nakajima, S., Hayashi, Y.-Y., \& Abe, Y. 1992, JAtS, 49, 2256

O'Gorman, P. A., \& Schneider, T. 2008, JCli, 21, 3815

Pierrehumbert, R., Brogniez, H., \& Roca, R. 2007, in The Global Circulation of the Atmosphere, ed. T. Schneider, \& A. H. Sobel (Princeton, NJ: Princeton Univ. Press)

Pierrehumbert, R., \& Gaidos, E. 2011, ApJL, 734, L13

Pierrehumbert, R. T. 2002, Natur, 419, 191

Pierrehumbert, R. T. 2011, Principles of Planetary Climate (Cambridge: Cambridge Univ. Press)

Rennó, N. O., Stone, P. H., \& Emanuel, K. A. 1994, JGRD, 99, 17001

Trenberth, K. E. 1997, JCli, 10, 2796

Wolf, E., \& Toon, O. 2015, JGRD, 120, 5775

Wordsworth, R., \& Pierrehumbert, R. 2014, ApJL, 785, L20

Wordsworth, R. D., Forget, F., Selsis, F., et al. 2011, ApJL, 733, L48

Wordsworth, R. D., \& Pierrehumbert, R. T. 2013, ApJ, 778, 154

Yang, J., Boué, G., Fabrycky, D. C., \& Abbot, D. S. 2014, ApJL, 787, L2 